\title{
Scaling limits of random walk bridges conditioned to avoid a finite set
}

\author{
Kôhei UCHIYAMA \\ Department of Mathematics, Tokyo Institute of Technology \\ Oh-okayama, Meguro Tokyo 152-8551
}

running head: Scaling limits of random walk bridges

key words: random walk on the integer lattice; conditioned to avoid a set; third moment; functional limit theorem; killing on a finite set; tightness of pinned walk; tunneling.

AMS Subject classification (2010): Primary 60G50, Secondary 60J45.

\begin{abstract}
This paper concerns a scaling limit of a one-dimensional random walk $S_{n}^{x}$ started from $x$ on the integer lattice conditioned to avoid a non-empty finite set $A$, the random walk being assumed to be irreducible and have zero mean. Suppose the variance $\sigma^{2}$ of the increment law is finite. Given positive constants $b, c$ and $T$ we consider the scaled process $S_{[t N]}^{b_{N}} / \sigma \sqrt{N}, 0 \leq t \leq T$ started from a point $b_{N} \approx b \sqrt{N}$ conditioned to arrive at another point $\approx-c \sqrt{N}$ at $t=T$ and avoid $A$ in between and discuss the functional limit of it as $N \rightarrow \infty$. We show that it converges in law to a continuous process if $E\left[\left|S_{1}\right|^{3} ; S_{1}<0\right]<\infty$. If $E\left[\left|S_{1}\right|^{3} ; S_{1}<0\right]=\infty$ we suppose $P\left[S_{1}<u\right]$ to vary regularly as $u \rightarrow-\infty$ with exponent $-\beta, 2 \leq \beta \leq 3$ and show that it converges to a process which has one downward jump that clears the origin if $\beta<3$; in case $\beta=3$ there arises the same limit process as in case $E\left[\left|S_{1}\right|^{3} ; S_{1}<0\right]<\infty$. In case $\sigma^{2}=\infty$ we consider the special case when $S_{1}$ belongs to the domain of attraction of a stable law of index $1<\alpha<2$ having no negative jumps and obtain analogous results.
\end{abstract}

\section{Introduction}

Let $S_{n}, n=0,1,2 \ldots$ be a random walk on $\mathbb{Z}$ started at the origin, namely $S_{0}=0$ and $S_{n+1}-S_{n}$ are i.i.d. random variables taking values in the integer lattice $\mathbb{Z}$. Let $\left(S_{n}\right)$ be defined on a probability space $(\Omega, \mathcal{F}, P)$ and suppose that $\left(S_{n}\right)$ is irreducible and

$$
E S_{1}=0
$$

Let $\sigma$ be the variance of the step variable: $\sigma=\left(E\left[\left|S_{1}\right|^{2}\right]\right)^{1 / 2}$. We consider the both of cases $\sigma<\infty$ and $\sigma=\infty$, but usually suppose $\sigma<\infty$ unless the contrary is stated explicitly when we discuss the problem for the case $\sigma=\infty$. In [14] the present author obtained a precise asymptotic form of transition probability of the walk $S$ killed on a finite nonempty set $A$ (in case $\sigma<\infty$ ). In the present paper we are interested in the behaviour of $S_{k}^{b_{N}}:=S_{k}+b_{N}, k=1, \ldots, N$, for large $N$, conditioned on the events $S_{k}^{b_{N}} \notin A$ for $k \leq N$ and $S_{N}^{b_{N}}=-b_{N}$ where $b_{N} \approx b \sqrt{N}, b>0$. In [14] it is observed that if $E\left[\left|S_{1}\right|^{3} ; S_{1}<0\right]<\infty$, then 
the walk thus conditioned "continuously" transits from the positive half line to the negative half still avoiding $A$, while if $E\left[\left|S_{1}\right|^{3} ; S_{1}<0\right]=\infty$ it clears $A$ by one "long jump". In this paper we observe that this is reflected to the scaling limit. In case $E\left[\left|S_{1}\right|^{3} ; S_{1}<0\right]=\infty$ we suppose that $P\left[S_{1}<u\right]$ is regularly varying as $u \rightarrow-\infty$ with index $-\beta, 2 \leq \beta \leq 3$. Then we prove that the scaled process converges in law and the limit process is continuous in the former case; in the latter case it has exactly one downward jump if $\beta<3$ while the limit process agrees with that of the former case if $\beta=3$. In case $\sigma=\infty$ analogous results are given for the special case when $S_{1}$ belongs to the domain of attraction of a stable law with exponent $1<\alpha<2$ having no negative jumps.

There are a lot of works dealing with various problems concerning random walks on the real line conditioned to avoid a finite set or a half line. To mention among them only those studying the functional limit theorems, the finite set case are studied by [1] and [10], whereas for the half line we have a long list of papers for which the readers are referred to [3], 6] where brief descriptions of them are found.

\section{Statements of results}

Supposing $\sigma<\infty$ we first describe the processes arising in the limit of the random walk bridges conditioned to avoid $A$, and then state the convergence results. The case $\sigma=\infty$ will be discussed separately after that. There appear some processes in the limit, which we suppose to be given in the same probability space as $\left(S_{n}\right)$.

Limit processes. Let $W_{t}, t \geq 0$ be a standard linear Brownian motion started at $0, Y_{t}^{x}$, $x, t \geq 0$ a 3 -dimensional Bessel process started at $x$, and $Y_{s}^{x, y, t}(0 \leq s \leq t, x \geq 0, y \geq 0)$ the Bessel bridge of length $t$ joining $x$ and $y$ obtained by conditioning $Y^{x}$. The processes $Y^{x}, x \geq 0$ are supposed to be independent of $W$. For $x \in \mathbb{R}$ we write $W_{t}^{x}$ for $W_{t}+x$ and let $\sigma_{0}^{x, W}$ be the first passage time of zero by $\left(W_{t}^{x}\right)$.

Put

$$
\mathfrak{g}_{t}(x)=\frac{1}{\sqrt{2 \pi t}} e^{-x^{2} / 2 t}, \quad \mathfrak{g}_{t}^{0}(x, y)=\mathfrak{g}_{t}(y-x)-\mathfrak{g}_{t}(x+y)
$$

and

$$
\rho_{t}(x)=\frac{|x|}{t} \mathfrak{g}_{t}(x)
$$

For each constants $T>0$ and $c>0, Y_{t}^{0, c, T}, 0 \leq t \leq T$ is a Markov process with transition law

$$
\begin{gathered}
\frac{P\left[Y_{t}^{0, c, T} \in d z\right]}{d z}=\frac{\rho_{t}(z) \mathfrak{g}_{T-t}^{0}(z, c)}{\rho_{T}(c)}, \\
\frac{P\left[Y_{t}^{0, c, T} \in d z \mid Y_{s}^{0, c, T}=w\right]}{d z}=\frac{\mathfrak{g}_{t-s}^{0}(w, z) \mathfrak{g}_{T-t}^{0}(z, c)}{\mathfrak{g}_{T-s}^{0}(w, c)}
\end{gathered}
$$

(cf. [11, Section VI.3]). In the sequel the letters $b, c$ and $T$ denote positive constants.

TRANSIT MADE BY CREEPING. Writing $\tau=\sigma_{0}^{b, W}$ we define the time-inhomogeneous process $X_{t}^{b, c, T}, 0 \leq t \leq T$ by

$$
X_{t}^{b, c, T}= \begin{cases}W_{t}^{b} & 0 \leq t \leq \sigma_{0}^{b, W} \\ -Y_{t-\tau}^{0, c, T-\tau} & \tau=\sigma_{0}^{b, W}<t \leq T\end{cases}
$$


on the event $\tau<T$. The process $X^{b, c, T}$ conditioned on $\{\tau<T\}$ is a Markov process on $\mathbb{R}$ (inhomogeneous in time) whose transition probability density $q(s, x ; t, y)$ is described below. (See (4.2) for the finite dimensional distribution.) Note that if $\tilde{W}$ is a linear Brownian motion independent of $W$, the Bessel bridge $Y_{t-\tau}^{0, c, T-\tau}$ in (2.2) can be substituted for by $\tilde{W}_{T-t}^{c}$ conditional on $\sigma_{0}^{c, \tilde{W}}=T-\tau$, the two processes having the same law (cf. e.g., [11, XI(3.12)]), provided the event $\{\tau<T\}$ conditioning $X_{t}^{b, c, T}$ is accordingly replaced by $\tau+\sigma_{0}^{c, \tilde{W}}=T$.

For $0<s<t<T$,

$$
q(s, x ; t, y)= \begin{cases}\frac{\mathfrak{g}_{t-s}^{0}(x, y) \rho_{T-t}(y+c)}{\rho_{T-s}(x+c)} & x \geq 0, y>0, \\ \frac{\rho_{t-s}(x-y) \mathfrak{g}_{T-t}^{0}(y,-c)}{\rho_{T-s}(x+c)} & y \leq 0 \leq x, \\ \frac{\mathfrak{g}_{t-s}^{0}(x, y) \mathfrak{g}_{T-t}^{0}(y,-c)}{\mathfrak{g}_{T-s}^{0}(x,-c)} & x<0, y \leq 0, \\ 0 & x<0<y,\end{cases}
$$

and for $0<t<T$,

$$
q(0, b ; t, x)=q(t, x ; T,-c)= \begin{cases}\frac{\mathfrak{g}_{t}^{0}(b, x) \rho_{T-t}(x+c)}{\rho_{T}(b+c)} & 0 \leq x, \\ \frac{\rho_{t}(b-x) \mathfrak{g}_{T-t}^{0}(x,-c)}{\rho_{T}(b+c)} & x<0 .\end{cases}
$$

REMARK 1. (a) The semi group property of $q(s, x ; t, y)$ can be directly ascertained by using the relations

$$
\int_{0}^{\infty} \rho_{t}(z) \mathfrak{g}_{s}^{0}(z, y) d z=\rho_{t+s}(y) \quad \text { and } \quad \int_{0}^{t} \rho_{t-s}(x) \rho_{s}(y) d s=\rho_{t}(x+y) \quad(x, y>0) .
$$

(e.g., to see $\int_{-\infty}^{\infty} q(0, b ; t, x) d x=1$ write $\rho_{t}(b-x)=\int_{0}^{t} \rho_{t-s}(-x) \rho_{s}(b) d s$ and similarly for $\left.\rho_{T-t}(x+c)\right)$. The former one says that the family $\left(\rho_{t}(z) d z\right)_{t>0, x>0}$ constitutes the entrance law for the semigroup $\mathfrak{g}_{t}^{0}(x, y) d y$ (cf., e.g., [11]) and the latter follows from the fact that $\rho$. $(y-x), y>x$ is the transition density of the passage time process $\left(\sigma_{x}^{0, W}\right)_{x \geq 0}([9])$.

(b) The process $X^{b, c, T}$ defined above can be obtained as a normalized limit of Brownian bridge killed at rate $\lambda \ell(t)$ as $\lambda \rightarrow \infty$, where $\ell(t)$ is the Brownian local time at zero (see Appendix (A)).

(c) Although the limit processes are described by means of the 3-dimensional Bessel process, one may think that there should naturally appear the Brownian meander. The bridges of the two processes have the same law which can be described by the Brownian motion killed on hitting zero together with the entrance law for it as is displayed in (2.1). (See also Remark 3.)

Transit made By A JUmp. Let $2 \leq \beta<3$. Put for $t>0$ and $y<0<x$,

$$
J_{t}(x, y)=\int_{0}^{t} d s \int_{0}^{\infty} \mathfrak{g}_{s}^{0}(x, w) d w \int_{0}^{\infty} \frac{\beta}{(w+z)^{1+\beta}} \mathfrak{g}_{t-s}^{0}(-z, y) d z
$$


(see Remark 2 below for the integrability). Let $\tau$ and $\eta$ be positive random variables dependent on $W^{b}$ with the conditional law

$$
\begin{array}{r}
P\left[\tau \in d t, \eta \in d z \mid \mathcal{F}_{t}^{W, b}\right]=\frac{\beta \mathfrak{g}_{T-t}^{0}(-z,-c)}{\left(W_{t}^{b}+z\right)^{1+\beta}} \cdot \frac{\mathbf{1}\left(\sigma_{0}^{b, W}>t\right) d t d z}{J_{T}(b,-c)}, \\
0<t<T, z>0 .
\end{array}
$$

where $\mathcal{F}_{t}^{W, b}$ is the $\sigma$-field on $\Omega$ generated by $W_{s}^{b}, s \leq t$. Note that

$$
E\left[\int_{0}^{T} d t \int_{0}^{\infty} \frac{\beta \mathfrak{g}_{T-t}^{0}(-z,-c)}{\left(W_{t}^{b}+z\right)^{1+\beta}} \mathbf{1}\left(\sigma_{0}^{b, W}>t\right) d z\right] \frac{1}{J_{T}(b,-c)}=1
$$

and $\tau$ is an $\mathcal{F}_{t}^{W, b}$-stopping time taking values in $(0, T)$ a.s. Let the Bessel processes $Y^{x}, x \geq 0$ be independent of $(\tau, \eta)$ as well as of $W$. Define

$$
\breve{X}_{t}^{b, c, T}= \begin{cases}W_{t}^{b} & 0 \leq t<\tau \\ -Y_{t-\tau}^{\eta, c, T-\tau} & \tau \leq t<T .\end{cases}
$$

Then $\breve{X}_{t}^{b, c, T}, t \leq T$ is a Markov process on $\mathbb{R} \backslash\{0\}$ and its transition probability is given by replacing $\rho$ by $J$ in (2.3) and (2.4): For $0<s<t<T$,

$$
\breve{q}(s, x ; t, y)= \begin{cases}\frac{\mathfrak{g}_{t-s}^{0}(x, y) J_{T-t}(y,-c)}{J_{T-s}(x,-c)} & x \geq 0, y>0 \\ \frac{J_{t-s}(x, y) \mathfrak{g}_{T-t}^{0}(y,-c)}{J_{T-s}(x,-c)} & y \leq 0 \leq x, \\ \frac{\mathfrak{g}_{t-s}^{0}(x, y) \mathfrak{g}_{T-t}^{0}(y,-c)}{\mathfrak{g}_{T-s}^{0}(x,-c)} & x<0, y \leq 0, \\ 0 & x<0<y,\end{cases}
$$

and for $0<t<T$,

$$
\breve{q}(0, b ; t, x)=\breve{q}(t, x ; T,-c)= \begin{cases}\frac{\mathfrak{g}_{t}^{0}(b, x) J_{T-t}(x,-c)}{J_{T}(b,-c)} & 0 \leq x, \\ \frac{J_{t}(b, x) \mathfrak{g}_{T-t}^{0}(x,-c)}{J_{T}(b,-c)} & x<0 .\end{cases}
$$

REMARK 2. The integrability of the repeated integral defining $J_{t}$ reduces to that of $\int_{0}^{1} d z \int_{0}^{1} z w(z+w)^{-1-\beta} d w$ which is finite if and only if $\beta<3$.

Scaling limits. Let $\left(S_{n}\right)$ be the random walk on $\mathbb{Z}$ as specified in Introduction. Let

$$
S_{n}^{x}=x+S_{n} \quad(x \in \mathbb{Z})
$$

and write $\sigma_{D}^{x}$ for the first hitting time of a non-empty set $D \subset \mathbb{Z}$ by $S^{x}$ :

$$
\sigma_{D}^{x}=\inf \left\{n \geq 1: S_{n}^{x} \in D\right\} .
$$

We shall write $S_{\sigma_{D}}^{x}$ for $S_{\sigma_{D}^{x}}^{x}$. Let $b$ and $c$ be positive constants, and $b_{N}$ and $c_{N}, N=1,2, \ldots$ two sequences of integers such that as $N \rightarrow \infty$

$$
b_{N} \sim b \sqrt{\sigma^{2} N} \text { and } \quad c_{N} \sim c \sqrt{\sigma^{2} N}
$$


(provided $\sigma^{2}<\infty$ ), and define the scaled process

$$
X^{(N)}=\text { the linear interpolation of }\left(S_{k}^{b_{N}} / \sqrt{\sigma^{2} N}: k=0,1,2, \ldots\right) \text {, }
$$

where $\sim$ means that the ratio of two sides of it approaches 1 . Let $T$ be an arbitrarily given positive constant and $A$ a non-empty finite subset of $\mathbb{Z}$. We are concerned with the law of $X^{(N)}$ under the condition that

$$
\sigma_{A}^{b_{N}}>N T \quad \text { and } \quad S_{\lfloor N T\rfloor}^{b_{N}}=-c_{N}
$$

namely the walk $S^{b_{N}}$ visits $-c_{N}$ at time $\lfloor N T\rfloor$ without entering $A(\lfloor\cdot\rfloor$ stands for the integer part). We suppose that $P\left[\sigma_{\{y\}}^{x}<\infty\right]>0$ for some, then all, $x>\max A$ and $y<\min A$ so that the probability of event (2.8) is positive for all sufficiently large $N$ if the walk is further supposed to be temporally aperiodic (which is not restrictive for the present problem). In what follows the probability of (2.8) is tacitly supposed to be positive when the conditioning on (2.8) is considered.

Let $F$ be the distribution function of $S_{1}: F(u)=P\left[S_{1} \leq u\right], u \in \mathbb{R}$.

Theorem 1. Suppose that $E\left[\left|S_{1}\right|^{3} ; S_{1}<0\right]<\infty$ or $F(u)$ is regularly varying with index -3 as $u \rightarrow-\infty$. Then the law of $\left(X_{t}^{(N)}: 0 \leq t \leq T\right)$ conditioned on event (2.8) converges to the law of $\left(X_{t}^{b,-c, T}\right)_{0 \leq t \leq T}$ conditioned on $\sigma_{0}^{b, W}<\bar{T}$ relative to the uniform topology of $C([0, T], \mathbb{R})$.

Theorem 2. Suppose that $F(u)$ is regularly varying with index $-\beta$ as $u \rightarrow-\infty, 2 \leq \beta<3$. Then the law of $\left(X_{t}^{(N)}: 0 \leq t \leq T\right)$ conditioned on event (2.8) converges to the law of $\left(\breve{X}_{t}^{b,-c, T}\right)_{0 \leq t \leq T}$ relative to the Skorohod topology of $D([0, T], \mathbb{R})$.

Here we briefly explain how the two types of limit processes emerge in Theorems 1 and 2 and thereby indicate a crucial point in question. For $N$ large the way $S^{b_{N}}$ enters the half line $(-\infty, 0]$ is virtually determined by

$$
H_{(-\infty, 0]}^{+\infty}(y)=\lim _{x \rightarrow \infty} P\left[S_{\sigma_{(-\infty, 0]}}^{x}=y\right], \quad y \leq 0,
$$

the hitting distribution of $(-\infty, 0]$ for the walk 'started at infinity'. The above limits exist and constitute a probability distribution on $(-\infty, 0]$ [12, Theorem 30.1]. It holds that $H_{(-\infty, 0]}^{+\infty}(y)$ is bounded above and below by positive multiples of $\int_{-\infty}^{y} F(u) d u$; in particular

$$
\sum_{y=-\infty}^{-1} H_{(-\infty, 0]}^{+\infty}(y)(-y)<\infty \quad \text { if and only if } E\left[\left|S_{1}\right|^{3} ; S_{1}<0\right]<\infty
$$

(cf. e.g., [14, (2.7)]). It is observed in [14] that $S^{b_{N}}$ conditioned on event (2.8) either comes near to $A$ but still avoids it or clears $A$ by one long jump that becomes indefinitely large as $N \rightarrow \infty$ according as the above infinite series is convergent or divergent. This would convince one that if the series are convergent there appears a continuous process in the limit. In case of the divergence the limit process may still be continuous and in order to ascertain it to be discontinuous we need to estimate the length of a typical jump to reveal whether it is comparable to the scale $\sqrt{N}$ so as to remain positive in the limit. (An answer to the question will be given in Lemma 3.2.)

Results for a random walk with $\sigma^{2}=\infty$. 
Let $1<\alpha<2$ and the distribution function of $S_{1}$ satisfy

$$
1-F(u) \sim L(u) / u^{\alpha} \text { and } F(-u) /[1-F(u)] \rightarrow 0 \quad \text { as } \quad u \rightarrow \infty,
$$

where $L(u)$ is a continuous positive function on $u \geq 0$ slowly varying at infinity. This condition is necessary and sufficient in order for the scaled process $S_{\lfloor n t\rfloor} / \lambda_{n}$ to converge in law to a strictly stable process of index $\alpha$ which has no negative jump, provided that the norming constants $\lambda_{n}$ are suitably chosen, which we may and do take so that $\lambda_{n}^{\alpha} / n L\left(\lambda_{n}\right) \rightarrow 1$. Let $a(x)$ denotes the potential function of the walk $S$ (defined in (3.1)). Then, corresponding to the equivalence relation (2.10) it holds that under the condition (2.11)

$$
\sum_{y=-\infty}^{-1} H_{(-\infty, 0]}^{+\infty}(y) a(y)<\infty \text { if and only if } \sum_{y=-\infty}^{-1}[a(y)]^{2} F(y)<\infty \text {. }
$$

Let $C^{+}$stand for the first sum in (2.12) so that $C^{+}<\infty \Longleftrightarrow \sum_{y=-\infty}^{-1}[a(y)]^{2} F(y)<\infty$. Then

$$
\lim _{x \rightarrow \infty} a(x)=C^{+}(\leq \infty)
$$

(Cf. [17, Theorem 2 and Corollary 2] for (2.12), (2.13).) Denote by $\left(Y_{t}\right)_{t \geq 0}$ the limiting stable process started from zero and put $Y_{t}^{x}=x+Y_{t}$. Let $\mathfrak{p}_{t}(x)$ be the probability density of $Y_{t}$ and $\mathfrak{p}_{t}^{0}(x, \cdot)$ the transition density of the stable process killed on hitting zero. For $x<0$ define $\rho_{t}(x)$ to be the density of the hitting time of $x$ for $Y$. Then

$$
\rho_{t}(x)=\frac{x}{t} \mathfrak{p}_{t}(-x) \quad \text { for } x>0
$$

(cf. [2, Corollary VII.3]). It follows that for $x<0$,

$$
\rho_{s+t}(-x)=\int_{-\infty}^{0} \rho_{t}(-z) \mathfrak{p}_{s}^{0}(z, x) d z
$$

(cf. Appendix (B)), saying that the family $\left(\rho_{t}(-x) d x: t>0, x<0\right)$ constitutes an entrance law for the transition semigroup of the processes $\left(Y_{t}^{x}\right)_{t \geq 0}, x<0$ killed on exiting from the negative half line $(-\infty, 0)$. Note that the transition function of this killed process agrees with $\mathfrak{p}_{t}^{0}(x, y)$ restricted to $x \vee y<0$.

Let $\left(X_{t}^{b,-c, T}\right)_{0 \leq t \leq T}$ be the process whose transition law is given as before by (2.3) and (2.4) but with $\mathfrak{p}_{t}^{0}$ in place of $\mathfrak{g}_{t}^{0}$ and similarly for $\breve{X}_{t}^{b, c, T}, t \leq T$. Instead of (2.6) let $b_{N} \sim b \lambda_{N}$ and $c_{N} \sim c \lambda_{N}$ and define the scaled process $X_{t}^{(N)}$ by

$$
X_{t}^{(N)}=S_{\lfloor t N\rfloor}^{b_{N}} / \lambda_{N}, \quad 0 \leq t \leq T .
$$

The probability of event (2.8) is supposed to be positive for $N$ large enough. Then the following theorems hold for $X_{t}^{(N)}$. It is noted that under (2.11)

$$
a(-y) \sim \kappa_{\alpha} y^{\alpha-1} / L(y) \quad \text { as } \quad y \rightarrow+\infty
$$

where $\kappa_{\alpha}=1 / \Gamma(\alpha) \Gamma(\alpha-1)$ (cf. [15, Lemma 3.1, $\operatorname{Eq}(9.2)$ ], [1, Lemma 3.3]), so that $C^{+}<\infty$ if $F(-y)=O\left(y^{-\beta}\right)$ with $\beta<2 \alpha-1$. 
Theorem 3. Suppose (2.11) to hold and that either $C^{+}<\infty$ or $F(u)$ is regularly varying with index $-2 \alpha+1$ as $u \rightarrow-\infty$. Then the law of $\left(X_{t}^{(N)}: 0 \leq t \leq T\right)$ conditioned on event (2.8) converges to the law of $\left(X_{t}^{b,-c, T}\right)_{0 \leq t \leq T}$ w.r.t. the Skorohod topology of $D([0, T], \mathbb{R})$.

Theorem 4. Suppose (2.11) to hold and that $F(u)$ is regularly varying as $u \rightarrow-\infty$ with index $-\beta, \alpha \leq \beta<2 \alpha-1$. Then the law of $\left(X_{t}^{(N)}: 0 \leq t \leq T\right)$ conditioned on event (2.8) converges to the law of $\left(\breve{X}_{t}^{b,-c, T}\right)_{0 \leq t \leq T}$ w.r.t. the Skorohod topology of $D([0, T], \mathbb{R})$.

REMARK 3. In Theorems 11 through 4 the scaled walk $X_{t}^{(N)}$ is considered under the conditional law given the two events given in (2.8). By the same token as what is mentioned in Remark 1(c) we can replace the first of it by $\sigma_{A}^{b_{N}}=\infty$ with the conclusion kept unaltered; in other words, if $P^{(A, b)}$ denotes the conditional law $P\left[\cdots \mid \sigma_{A}^{b_{N}}=\infty\right]$, the bridge $P^{(A, b)}\left[\cdots \mid X_{T}^{(N)}=-c_{N}\right]$ converges to the same limit laws as specified above.

In the next section we collect the known results concerning the walk $S^{x}$ killed on $A$ which are used in the proofs of Theorems 1 to 4, and prove some related lemmas, especially Lemma 3.2 mentioned above. The proofs of Theorems 1 and 2 are given in Sections 4.1 and 4.2, in which the tightness of the sequence of conditional laws of $X^{(N)}$ is verified and the finite dimensional distributions of limit processes are derived. Theorems 3 and 4 are proved in almost the same way as Theorems 1 and 2 and we do not provide full proofs of them except for some remarks and key lemmas that we give in Section 4.3 as well as Section 3 .

\section{$3 \quad$ Preliminary Results}

We present known results taken mainly from [12], [13] and [14] and prove some related results. In the rest of this paper the letters $x, y, z$ and $w$ always denote the integers representing states of the walk.

For a non-empty $B \subset \mathbb{Z}$ denote by $p_{B}^{n}(x, y)$ the transition probability of the walk $S_{n}$ killed upon entering $B$, which we define by

$$
p_{B}^{n}(x, y)=P\left[S_{n}^{x}=y, \sigma_{B}^{x}>n\right], \quad n=0,1,2, \ldots
$$

This entails $p_{B}^{n}(x, y)=0$ whenever $y \in B, n \geq 1$; and $p_{B}^{0}(x, y)=\delta_{x, y}$ even if $y \in B$, where $\delta_{x, y}$ equals unity if $x=y$ and zero if $x \neq y$ (recall that $\sigma_{B}^{x} \geq 1$ for every $x$ ). Put

$$
p(x)=P\left[S_{1}=x\right] \quad \text { and } \quad F(u)=P\left[S_{1} \leq u\right] \quad(u \in \mathbb{R}) .
$$

We suppose that the walk is (temporally) aperiodic, namely for every $x \in \mathbb{Z}$ there exists $n_{x} \geq 1$ such that $P\left[S_{n}=x\right]>0$ for $n \geq n_{x}$, which does not give rise to any loss of generality.

Let $a(x)$ be the potential function of the walk defined by

$$
a(x)=\lim _{n \rightarrow \infty} \sum_{k=0}^{n}\left[p^{k}(0)-p^{k}(-x)\right] .
$$

It holds that $a(x+1)-a(x) \rightarrow \pm 1 / \sigma^{2}$ as $x \rightarrow \pm \infty$, which implies

$$
a(x+z)-a(x)= \pm z\left\{1 / \sigma^{2}+o(1)\right\}
$$


with $o(1) \rightarrow 0$ as $x \rightarrow \pm \infty$ uniformly for $z$ with $(x+z) x>0$, and $a(x) /|x| \rightarrow 1 / \sigma^{2}$ as $|x| \rightarrow \infty$. (Cf. Theorem 28.1 and Theorem 29.2 of [12].) Let $g_{\{0\}}(x, y)=\sum_{n=0}^{\infty} p_{\{0\}}^{n}(x, y)$, the Green function of the walk killed on visiting the origin. We have the identity

$$
g_{\{0\}}(x, y)=a^{\dagger}(x)+a(-y)-a(x-y)
$$

where $a^{\dagger}(x)=a(x)+\delta_{0, x}$ [12, Proposition 29.4]. In what follows these relations will be used frequently and not noticed of their use. On putting

$$
\lambda(x)=a(x)-x / \sigma^{2}, x \in \mathbb{Z}
$$

it also holds [13, (2.9)], [14, (2.15)] that if $\sigma^{2}<\infty$,

$$
E\left[\lambda\left(S_{\sigma_{(-\infty, 0]}}^{x}\right)\right]=\lambda(x) \quad \text { for } \quad x \geq 1 .
$$

Let $A$ be a non-empty finite subset of $\mathbb{Z}$. For convenience of description we assume that $\max A=0$ (namely $0 \in A$ and $A \subset(-\infty, 0])$ and that

$$
P\left[\sigma_{\{y\}}^{x}<\sigma_{A}^{x}\right]>0 \text { for some } x>0 \text { and } y<\min A .
$$

In the following statements a constant $M>1$ is given arbitrarily in advance. In the square bracket at the head of each of them is indicated the proposition which the result is taken from. $C, C^{\prime}, C_{1}, \ldots$ etc. denote unimportant positive constants whose values may depend on $F$ and vary at different occurrences of them. Sometimes these constant depends on $M$ and possibly on $A$, in which case we write $C_{M}, C_{M, A}$, etc.

P0 [14, Remark 4 (Sect. 5), Theorem C (Sect. 4.2)] Uniformly for positive $x, y<$ $M \sqrt{n}$

$$
p_{(-\infty, 0]}^{n}(x, y)=p_{\{0\}}^{n}(x, y)\left\{1+O\left(y^{-1}+\lambda(x) x^{-1}\right)\right\} \quad \text { and } \quad p_{(-\infty, 0]}^{n}(x, y) \asymp x y / n^{3 / 2} .
$$

(Here and in the sequel $\asymp$ means that the ratio of two sides of it is bounded away from zero and infinity.)

In view of the first relation above $p_{\{0\}}^{n}(x, y)$ and $p_{A}^{n}(x, y)$ may be interchangeable in most of the arguments made later if $x y>0$, since the precision of estimates for small values of $x, y$ are irrelevant for the proofs of Theorems 1 and 2 .

P1 [13, Theorem 1.1]. (a) Uniformly for $x \in \mathbb{Z}$ and $y \in \mathbb{Z}$ subject to the constraints $-M \leq x \leq M \sqrt{n}$ and $-M \leq y \leq M \sqrt{n}$, as $n \rightarrow \infty$ and $(|x| \wedge|y|) / \sqrt{n} \rightarrow 0$

$$
p_{\{0\}}^{n}(x, y)=\frac{\sigma^{4} a^{\dagger}(x) a(-y)+x y}{\sigma^{2} n} \mathfrak{g}_{\sigma^{2} n}(y-x)\{1+o(1)\} .
$$

(b) As $x \wedge y \wedge n \rightarrow \infty$ under $x \vee y<M \sqrt{n}$

$$
p_{\{0\}}^{n}(x, y)=\mathfrak{g}_{\sigma^{2} n}^{0}(x, y)\{1+o(1)\} \text {. }
$$

(c) Whenever $1 \leq|x| \wedge|y|<\frac{1}{2}(|x| \vee|y|)$,

$$
p_{\{0\}}^{n}(x, y) \leq C_{M} \frac{\sqrt{n} \wedge|x| \wedge|y|}{(|x| \vee|y|)^{2}}
$$


[Here Spitzer's bound $p^{n}(x)=o\left(\sqrt{n} / x^{2}\right)(|x| / \sqrt{n} \rightarrow \infty)$ (cf. [12]) is also employed.]

(d) For all $n \neq 0, x \neq 0$ and $y$,

$$
p_{\{0\}}^{n}(x, y) \leq C|x y| / n^{3 / 2}
$$

in case $(|x| \vee|y|) / \sqrt{n} \rightarrow \infty$ the right side may be replaced by o(xy/n $\left.n^{3 / 2}\right)$.

P2 [14, Theorem 2]. If $E\left[\left|S_{1}\right|^{3} ; S_{1}<0\right]<\infty$, then

(a) $E\left[\lambda\left(S_{\sigma_{(-\infty, 0]}}^{x}\right)\right]=\lambda(x) \longrightarrow \sum_{z=0}^{\infty} H_{(-\infty, 0]}^{+\infty}(-z)\left[a(-z)+z / \sigma^{2}\right]<\infty \quad$ as $\quad x \rightarrow \infty$;

(a') $H_{(-\infty, 0]}^{+\infty}(-z)=P[\hat{Z}<-z] / E|\hat{Z}|=o\left(1 / z^{2}\right) ; \quad$ and

(b) as $x \wedge(-y) \wedge n \rightarrow \infty$ subject to the condition $x \vee(-y)<M \sqrt{n}$

$$
p_{A}^{n}(x, y) \sim C_{A}^{+} \rho_{\sigma^{2} n}(x+|y|),
$$

where $C_{A}^{+}$is some positive constant determined by $A$ and $F$ (see [14 for an explicit form).

P3 [14, Proposition 8]. For $-M \sqrt{n}<y<0<x<M \sqrt{n}$ and for $n$ large enough

$$
p_{A}^{n}(x, y) \geq c_{M}\left(1+\sum_{w=1}^{x \wedge|y|} p(-w) w^{3}\right) \frac{x+|y|}{n^{3 / 2}}
$$

where $c_{M}$ is a positive constant depending on $M$ as well as $F$ and $A$.

P4 [14, Corollary 7]. Under $-M \sqrt{n}<y<0<x<M \sqrt{n}$

$$
\begin{aligned}
& P\left[S_{\sigma_{(-\infty, 0]}}^{x}<-\eta \mid n<\sigma_{\{0\}}^{x}, S_{n}^{x}=y\right] \\
& \quad \longrightarrow\left\{\begin{array}{lll}
0 & \text { as } \eta \rightarrow \infty \\
1 & \text { as } x \wedge(-y) \rightarrow \infty \text { for each } \eta>0 & \text { if } E\left[|X|^{3} ; X<0\right]=\infty .
\end{array}\right.
\end{aligned}
$$

P5 [14, Corollary 3], [13, Propositions 2.2 and 2.3]. Uniformly for $x>0$ and $r>0$, as $R \rightarrow \infty$

$$
P\left[S_{\sigma_{[R, \infty)}}^{x}>R+r ; \sigma_{[R, \infty)}^{x}<\sigma_{(-\infty, 0]}^{x}\right] \leq r^{-1} x \times o(1)
$$

P6 [13, Theorem 1.4]. Let $h_{x}$ be the space-time distribution of the first entrance of $S^{x}$ into $(-\infty, 0]$, namely $h_{x}(n, y)=P\left[S_{\sigma_{(-\infty, 0]}}^{x}=y, \sigma_{(-\infty, 0]}^{x}=n\right] \quad(x \geq 1, y \leq 0)$. Then uniformly for $y \leq 0<x<M \sqrt{n}$, as $n \rightarrow \infty$

$$
h_{x}(n, y)=\frac{f^{+}(x) \mathfrak{g}_{n}(x)}{n} H_{(-\infty, 0]}^{+\infty}(y)\{1+o(1)\}+o\left(\frac{x}{n^{2}}\right) ;
$$

and there exists a positive constant $c$ such that whenever $y \leq 0, x \wedge n \geq 1$,

$$
h_{x}(n, y) \leq c H_{(-\infty, 0]}^{+\infty}(y)\left(\frac{1}{n} \wedge \frac{1}{x \sqrt{n}}\right)
$$

Here $H_{(-\infty, 0]}^{+\infty}$ is the probability law given in (2.9) and $f^{+}(x), x \geq 1$ is a positive multiple of the renewal function of the descending ladder height process with the multiplicative constant chosen so that $f^{+}(x) \sim x$ as $x \rightarrow \infty$. [Although (3.7) is restricted to $x>\sqrt{n}$ in [13], the extension to $x \leq \sqrt{n}$ is easily verified (cf. [14, Lemma 4.1] or proof of [15, Lemma 6.5].) ] 
Lemma 3.1. If $E\left[\left|S_{1}\right|^{3} ; S_{1}<0\right]<\infty$, then for some constant $C$,

$$
p_{\{0\}}^{n}(x, y) \leq C \frac{1}{y^{2} \vee n} \quad \text { whenever } y<0 \leq x .
$$

Proof. First consider the case $y<-2 \sqrt{n}$ and suppose that $n$ is even for convenience of description. In the decomposition

$$
p_{\{0\}}^{n}(x, y)=\sum_{k=1}^{n} \sum_{z=1}^{\infty} h_{x}(k,-z) p_{\{0\}}^{n-k}(-z, y)
$$

we break the double sum on the right into three parts:

$$
I:=\sum_{k=1}^{n} \sum_{z \leq|y| / 2}, \quad J_{\leq n / 2}:=\sum_{k=1}^{n / 2} \sum_{z>|y| / 2} \text { and } J_{>n / 2}:=\sum_{k=1+n / 2}^{n} \sum_{z>|y| / 2} \text { (say). }
$$

By P1(c) it follows that for $z \leq|y| / 2, p_{\{0\}}^{n-k}(-z, y) \leq C(z \wedge \sqrt{n-k}) / y^{2} \leq C^{\prime} z / y^{2}$, so that uniformly for $x>0$,

$$
I \leq C^{\prime} \sum_{0<z \leq|y| / 2} H_{(-\infty, 0]}^{x}(-z) \frac{z}{y^{2}} \leq C^{\prime \prime} / y^{2}
$$

where for the last inequality we use the assumption $E\left[\left|S_{1}\right|^{3} ; S_{1}<0\right]<\infty$ (see P2(a)). Observe that $\sup _{k \leq n / 2} p_{\{0\}}^{n-k}(-z, y)$ is dominated by a constant multiple of $\left[\sqrt{n} /(y+z)^{2}\right] \wedge n^{-1 / 2}$, whose sum over $z>|y| / 2$ is bounded by a constant. Using this and $\mathbf{P 2}\left(\mathrm{a}^{\prime}\right)$ in turn we deduce that

$$
J_{\leq n / 2} \leq \sum_{z>|y| / 2} H_{(-\infty, 0]}^{x}(-z) \sup _{k \leq n / 2} p_{\{0\}}^{n-k}(-z, y) \leq C \sup _{z>|y| / 2} H_{(-\infty, 0]}^{+\infty}(-z)=o\left(1 / y^{2}\right) .
$$

By the second half of $\mathbf{P} 6$ we have $h_{x}(k,-z) \leq C n^{-1} H_{(-\infty, 0]}^{+\infty}(-z)$ for $k>n / 2$, and hence, employing P2(a') again,

$$
J_{>n / 2} \leq \frac{o\left(1 / y^{2}\right)}{n} \sum_{k=n / 2}^{n} \sum_{z>|y| / 2} p_{\{0\}}^{n-k}(-z, y)=o\left(1 / y^{2}\right)
$$

Thus the bound of the lemma is obtained if $|y| \geq 2 \sqrt{n}$.

For $0 \leq y<2 \sqrt{n}$, we break the outer sum at $n / 2$ in the right side expression of (3.8). Use (3.7) together with $g_{\{0\}}(-z, y) \leq g_{\{0\}}(-z,-z) \leq C a(-z)(z>0)$ for the sum over $k>n / 2$, which is then evaluated to be at most a constant multiple of $n^{-1} \sum_{z=1}^{\infty} H_{(-\infty, 0]}^{\infty}(-z) z \leq C / n$. The same bound is obtained for the other sum by using $p^{n-k}(-z, y) \leq C z y / n^{3 / 2}$ valid for $k \leq n / 2($ cf. $\mathbf{P} \mathbf{1}(\mathrm{d}))$.

Lemma 3.2. Suppose that $F$ is regularly varying at $-\infty$ with index $-\beta, \beta \in[2,3]$. Then for each $M>1$ the following hold uniformly for $x, y$ satisfying $-M \sqrt{n}<y<0<x<M \sqrt{n}$.

(a) If $2 \leq \beta<3$

$$
P\left[S_{\sigma_{(-\infty, 0]}}^{x} \leq-\varepsilon(x \wedge|y|) \mid S_{n}^{x}=y, \sigma_{A}^{x}>n\right] \rightarrow 1 \quad \text { as } x \wedge(-y) \wedge \varepsilon^{-1} \rightarrow \infty .
$$


(b1) If $2<\beta<3$,

$$
P\left[S_{\sigma_{(-\infty, 0]}}^{x}<-K(x \wedge|y|) \mid S_{n}^{x}=y, \sigma_{A}^{x}>n\right] \rightarrow 0 \quad \text { as } x \wedge(-y) \wedge K \rightarrow \infty .
$$

(b2) If $\beta=2$, (3.9) holds for $x, y$ satisfying $x \wedge|y| \geq M^{-1} \sqrt{n}$ in addition.

(c) If $\beta=3$ and $E\left[\left|S_{1}\right|^{3} ; S_{1}<0\right]=\infty$, then for each $\varepsilon>0$

$$
P\left[-\varepsilon(x \wedge|y|)<S_{\sigma_{(-\infty, 0]}}^{x}<-1 / \varepsilon \mid S_{n}^{x}=y, \sigma_{A}^{x}>n\right] \rightarrow 1 \quad \text { as } x \wedge|y| \rightarrow \infty .
$$

Proof. Let $F$ be regularly varying as is assumed in the lemma. As $m \rightarrow \infty$ by Karamata's theorem

$$
\sum_{w=1}^{m} p(-w) w^{3} \sim \frac{\beta}{3-\beta} m^{3} F(-m) \quad(\beta \neq 3) .
$$

Note that $P\left[S_{\sigma_{(-\infty, 0]}}^{x} \geq-\varepsilon(x \wedge|y|), S_{n}^{x}=y, \sigma_{A}^{x}>n\right]$ is non-increasing in $A$ and then observe that (a) follows if we show that as $x \wedge(-y) \wedge n \rightarrow \infty$

$$
\frac{\sum_{k=1}^{n} \sum_{w=1}^{\infty} \sum_{-\varepsilon(x \wedge|y|) \leq z<0} p_{(-\infty, 0]}^{k-1}(x, w) p(z-w) p_{\{0\}}^{n-k}(z, y)}{(x \vee|y|) n^{-3 / 2} \sum_{w=1}^{x \wedge|y|} p(-w) w^{3}} \rightarrow 0,
$$

owing to P3. As before we split the outer summation of the numerator at $k=n / 2$. Employing the obvious inequality $p_{(-\infty, 0]}^{k-1} \leq p_{\{0\}}^{k-1}$ together with $\mathbf{P} \mathbf{1}(\mathrm{d})$ we infer that the sums over $k \leq n / 2$ and $k>n / 2$ are dominated by constant multiples of

$$
|y| n^{-3 / 2} \sum_{-\varepsilon(x \wedge|y|) \leq z<0} \sum_{w=1}^{\infty} g_{\{0\}}(x, w) p(z-w)|z|
$$

and

$$
x n^{-3 / 2} \sum_{-\varepsilon(x \wedge|y|) \leq z<0} \sum_{w=1}^{\infty} w p(z-w) g_{\{0\}}(z, y),
$$

respectively. We have the bound $g_{\{0\}}(x, y) \leq C(|x| \wedge|y|)$ for all $x$ and $y$ with $x y \neq 0$. On putting $m=\lfloor\varepsilon(x \wedge|y|)\rfloor$ and substituting from $g_{\{0\}}(x, w) \leq C|w|$ and $g_{\{0\}}(z, y) \leq C|z|$ an elementary computation verifies that both of the sums of the above double series are dominated by a constant multiple of $\sum_{w=1}^{\infty} \sum_{z=1}^{m} p(-z-w) z w$ which is at most

$$
\frac{1}{2} \sum_{u=1}^{2 m} \sum_{v=0}^{u} p(-u)\left(u^{2}-v^{2}\right)+\frac{1}{2} m^{2} \sum_{m}^{\infty} p(-w) w \leq C m^{3} F(-m) .
$$

Since $m^{3} F(-m) \sim \varepsilon^{3-\beta}(x \wedge|y|)^{3} F(-x \wedge|y|)$, this together with (3.10) shows (3.11) if $\beta<3$.

As for (b1) and (c) we show shortly that if $\beta>2$, then for all $m$ large enough

$$
\sum_{k=1}^{n} \sum_{z<-m} p_{\{0\}}^{k-1}(x, w) p(z-w) p_{\{0\}}^{n-k}(z, y) \leq \frac{C(x \wedge|y|) m^{2} F(-m)(x \vee|y|)}{n^{3 / 2}}
$$

If $\beta \neq 3$, on taking $m=\lfloor K(x \wedge|y|)\rfloor$ (b1) is immediate from this in view of (3.10). If $\beta=3$, then $w^{3} F(-w)$ is slowly varying and it follows that $\sum_{1}^{m} w^{3} p(-w) \sim 3 \sum_{1}^{m} w^{2} F(-w)$ and

$$
\frac{m^{3} F(-m)}{\sum_{1}^{m} w^{3} p(-w)} \rightarrow 0 \quad \text { as } \quad m \rightarrow \infty .
$$


On using P3 as before this together with (3.15) shows that the conditional probability of the event $S_{\sigma_{(-\infty, 0]}}^{x} \leq-\varepsilon(x \wedge|y|)$ tends to zero. (c) now follows from P4.

The proof of (3.15) is similar to the one given above for (3.11). What we should evaluate are

$$
\begin{aligned}
& \sum_{z<-m} \sum_{w=1}^{\infty} g_{\{0\}}(x, w) p(z-w) p_{\{0\}}^{n}(z, y) ; \text { and } \\
& \sum_{z<-m} \sum_{w=1}^{\infty} p_{\{0\}}^{n}(x, w) p(z-w) g_{\{0\}}(z, y)
\end{aligned}
$$

instead of (3.12) and (3.13), respectively. By $g_{\{0\}}(x, w) \leq C x$ it plainly follows that

$$
\sum_{w=1}^{\infty} g_{\{0\}}(x, w) p(z-w) \leq C^{\prime} x F(z),
$$

and if $\beta>2$ the sum of the first double series in $(\underline{3.16})$ is at most a constant multiple of

$$
x \sum_{z<-m} F(z) p_{\{0\}}^{n}(z, y) \leq C x|y| m^{2} F(-m) n^{-3 / 2} .
$$

Noting that $\sum_{w=1}^{\infty} w p(z-w)=\sum_{w=1}^{\infty} F(z-w) \sim(\alpha-1)^{-1} F(z)|z|$ as $z \rightarrow-\infty$ a similar computation leads to the same upper bound for the second one. This shows (3.15), since $|x y|=(x \wedge|y|)(x \vee|y|)$.

It remains to verify (b2). If $\beta=2$, the upper bound (3.18) is not valid but in (b2) it is supposed that $x \wedge|y|>\sqrt{n} / M$ so that if $m=K(x \wedge|y|)(K>1)$, then $p_{\{0\}}^{n}(z, y) \leq C \sqrt{n} / z^{2}$ for $z<-m$ and $|y|<M \sqrt{n}$ owing to $\mathbf{P 1}(\mathrm{c})$, and we obtain instead of (3.18)

$$
x \sum_{z<-m} F(z) p_{\{0\}}^{n}(z, y) \leq C^{\prime} x \sqrt{n} \sum_{z<-m} F(z) / z^{2} \leq \frac{C_{M} x F(-m)}{K} \leq C_{M}(x \vee|y|) F(-m) .
$$

Observing that the second sum in (3.16) admits the same upper bound we conclude (b2) to be true since $F(-m) \leq C_{M}^{\prime} K^{-2}(x \wedge|y|)^{3} F(-x \wedge|y|) / n^{3 / 2}$ for $n$ large enough.

Corollary 3.1. Let $F$ be as in the preceding lemma and suppose $E\left[\left|S_{1}\right|^{3} ; S_{1}<0\right]=\infty$ if $\beta=3$. Then for each $M>1$, uniformly for $x$, y satisfying $-M \sqrt{n}<y<0<x<M \sqrt{n}$,

(a) $P\left[S_{k}^{x}<0\right.$ for $\left.\sigma_{(-\infty, 0]}^{x} \leq k \leq n \mid S_{n}^{x}=y, \sigma_{A}^{x}>n\right] \rightarrow 1 \quad(x \wedge|y| \rightarrow \infty)$,

(b) if $2 \leq \beta<3$,

$$
P\left[\left|S_{\sigma_{(-\infty, 0]}}^{x}\right| \wedge S_{\sigma_{(-\infty, 0]}-1}^{x}>\varepsilon(x \wedge|y|) \mid S_{n}^{x}=y, \sigma_{A}^{x}>n\right] \rightarrow 1 \quad\left(x \wedge|y| \wedge \varepsilon^{-1} \rightarrow \infty\right),
$$

(c) if $\beta=3$, then for each $\varepsilon>0$

$$
P\left[\left|S_{\sigma_{(-\infty, 0]}}^{x}\right| \vee S_{\sigma_{(-\infty, 0]}-1}^{x}<\varepsilon(x \wedge|y|) \mid S_{n}^{x}=y, \sigma_{A}^{x}>n\right] \rightarrow 1 \quad(x \wedge|y| \rightarrow \infty) .
$$

Proof. The first relation follows from $\mathbf{P} 4$ and $\mathbf{P 0}$, the latter asserting $p_{\{0\}}^{n}(z, y) / p_{[0, \infty)}^{n}(z, y) \rightarrow$ 1 as $z \vee y \rightarrow-\infty$. By duality relations the rest follows from Lemma 3.2 .

Corresponding lemmas for the case $\sigma^{2}=\infty$

Here we suppose that (2.11) is satisfied and prove Lemmas 3.3 and 3.4 below that correspond to Lemmas 3.1 and 3.2 , respectively, and will be used for the proofs of Theorems 3 and 4. We shall use the following large deviation estimate:

$$
p^{n}(x) \leq C\left\{1 \wedge\left[n L(|x|) /|x|^{\alpha}\right]\right\} / \lambda_{n}
$$


(cf. [4). We also have

$$
H_{(-\infty, 0]}^{x}\{a\}=a(x), \quad x \geq 1
$$

valid if $E\left[S_{\sigma 0, \infty)}^{0}\right]=\infty$ always true under (2.11) (cf. [17, Corollary 1]). Recall $\lim _{x \rightarrow \infty} a(x)=$ $C^{+} \leq \infty$. Most of the results given below are based on [15] in which the transition function of the killed walk denoted by $Q_{A}^{n}(x, y)$ is defined slightly differently from but agrees with $p_{A}^{n}(x, y)$ whenever $y \notin A$.

Lemma 3.3. If $C^{+}<\infty$, then for some constant $C$,

$$
p_{\{0\}}^{n}(x, y) \leq C\left(\frac{L(|y|)}{(-y)^{\alpha}} \wedge \frac{1}{n}\right) \quad \text { whenever } y<0 \leq x .
$$

Proof. For $y<-2 \lambda_{n}$, the proof parallels to that of Lemma 3.1 with the help of the following bounds

$$
\begin{array}{cl}
p_{\{0\}}^{n}(x, y) \vee p_{\{0\}}^{n}(y, x) \leq C \frac{\left[|x|^{\alpha-1} / L(|x|)\right] \wedge\left[n / \lambda_{n}\right]}{|y|^{\alpha} / L(|y|)} & (1 \leq|x|<|y| / 2), \\
h_{x}(n, y) \leq C\left(\frac{1}{n} \wedge \frac{L(x)}{x^{\alpha}}\right) \frac{x}{\lambda_{n}} H_{(-\infty]}^{+\infty}(y) & (y<0<x)
\end{array}
$$

that follow immediately from Lemma 6.2 (combined with (3.19)) and Lemma 6.5(ii) of [15], respectively. As for $-2 \lambda_{n} \leq y<0$, in view of the identity $p_{\{0\}}^{n}(x, y)=p_{\{0\}}^{n}(-y,-x)$ what has been just proved yields the bound $p_{\{0\}}^{n}(x, y)<C / n$ for $x>2 \lambda_{n}$. For $x \leq 2 \lambda_{n}$, a better bound is obtained in [15, Proposition 2.3(i)] (applied to $p_{\{0\}}^{n}(-y,-x)$ ).

Lemma 3.4. Suppose $F$ is regularly varying at $-\infty$ with index $-\beta, \alpha \leq \beta \leq 2 \alpha-1$ in addition to (2.11). For each $M>1$ the following holds uniformly for $x, y$ satisfying $-M \lambda_{n}<y<0<x<M \lambda_{n}$.

$\left(\mathrm{a}^{*}\right)$ If $\alpha \leq \beta<2 \alpha-1$,

$$
P\left[S_{\sigma_{(-\infty, 0]}}^{x} \leq-\varepsilon(x \wedge|y|) \mid S_{n}^{x}=y, \sigma_{A}^{x}>n\right] \rightarrow 1 \quad \text { as } x \wedge(-y) \wedge \varepsilon^{-1} \rightarrow \infty .
$$

( $\left.\mathrm{b}^{*} 1\right)$ If $\alpha<\beta<2 \alpha-1$, under the additional constraint $x \vee|y| \leq M(x \wedge|y|)$

$$
P\left[S_{\sigma_{(-\infty, 0]}}^{x}<-K(x \wedge|y|) \mid S_{n}^{x}=y, \sigma_{A}^{x}>n\right] \rightarrow 0 \text { as } x \wedge(-y) \wedge K \rightarrow \infty .
$$

( $\left.\mathrm{b}^{*} 2\right)$ If $\beta=\alpha$, (3.23) holds for $x, y$ satisfying $x \wedge|y| \geq M^{-1} \lambda_{n}$ in addition.

$\left(\mathrm{c}^{*}\right)$ If $\beta=2 \alpha-1$ and $C^{+}=\infty$, for each $\varepsilon>0$

$$
P\left[-\varepsilon(x \wedge|y|)<S_{\sigma_{(-\infty, 0]}}^{x}<-1 / \varepsilon \mid S_{n}^{x}=y, \sigma_{A}^{x}>n\right] \rightarrow 1 \quad \text { as } x \wedge|y| \rightarrow \infty .
$$

The proof of the lemma proceeds parallel to that of Lemma 3.2 and we point out only main steps after stating the results from [15] needed for it. Put for $x>0$

$$
D_{n}(x)=\frac{a(x) \lambda_{n}}{n^{2}}+\frac{x}{n \lambda_{n}} .
$$

Instead of $\mathbf{P} 3$ it holds that if either $C^{+}<\infty$ or $F(x)$ is regularly varying as $x \rightarrow-\infty$, then

$$
p_{\{0\}}^{n}(x, y) \geq C_{M}^{-1}\left\{D_{n}(x) a^{\dagger}(-y)+D_{n}(-y) a^{\dagger}(x)\right\} \quad\left(-M \lambda_{n}<y \leq 0 \leq x<M \lambda_{n}\right)
$$


(the reversed inequality with $C_{M}^{-1}$ replaced by $C_{M}$ also holds), where $C_{M}$ is a positive constant (see Proposition 2.3(ii) of [15] as well as the comment given right after it). We also have

$$
p_{\{0\}}^{n}(x, w) \leq C D_{n}(x)\left\{a(-w) \wedge a\left(-\lambda_{n}\right)\right\} \quad\left(0<x<M \lambda_{n}, w>0\right)
$$

and its dual $p_{\{0\}}^{n}(z, y)=p_{\{0\}}^{n}(-y,-z) \leq C D_{n}(-y)\left\{a(z) \wedge a\left(-\lambda_{n}\right)\right\}\left(z<0,-M \lambda_{n}<y<0\right)$ (cf. [15, Lemma 6.1(i)]). Since $D_{n}(x) \leq C\left[x^{\alpha-1} / L(x)\right] \lambda_{n} / n^{2}$, (3.25) together with $p^{n}(x) \leq$ $C / \lambda_{n}$ entails

$$
p_{\{0\}}^{n}(x, y) \leq C \frac{|x y|^{\alpha-1} \lambda_{n}}{L(|x|) L(|y|) n^{2}} \quad(x y \neq 0) .
$$

Proof of $\left(\mathrm{a}^{*}\right)$. (3.11), (3.12) and (3.13) are modified in an obvious way according to (3.24), (3.26) and (3.25). Put $m=\lfloor\varepsilon(x \wedge|y|)\rfloor$ as before. Noting $g_{\{0\}}(x, y) \leq C[a(-|x|) \wedge a(-|y|)]$ $(x y \neq 0)$, we see first that $\sum_{w=1}^{\infty} \sum_{z=1}^{m} p(-z-w) a(-z) a(-w)$ is at most a constant multiple of

$$
\sum_{u=1}^{2 m} \sum_{v=0}^{u} \frac{p(-u)\left(u^{2}-v^{2}\right)^{\alpha-1}}{L(u+v) L(|u-v|)}+\frac{m^{\alpha}}{L(m)} \sum_{m}^{\infty} \frac{p(-w) w^{\alpha-1}}{L(w)} \leq C \frac{m^{2 \alpha-1} F(-m)}{L^{2}(m)},
$$

an estimate corresponding to (3.14), and then that $\left(\mathrm{a}^{*}\right)$ follows if we show that

$$
\frac{a(x) \vee a(-y)}{n^{2} / \lambda_{n}} \cdot \frac{m^{2 \alpha-1} F(-m)}{L^{2}(m)} / \frac{a(x) a(-y)}{n^{2} / \lambda_{n}} \rightarrow 0
$$

as $x \wedge(-y) \wedge \varepsilon^{-1} \rightarrow \infty$. Now let $F(-u) \sim L_{-}(u) / u^{\beta}(u \rightarrow \infty)$ for a slowly varying function $L_{-}$. Then for $\alpha \leq \beta<2 \alpha-1$ we have

$$
a(x) \sim C_{1} L_{-}(x) x^{2 \alpha-\beta-1} / L^{2}(x) \sim C_{1} x^{2 \alpha-1} F(-x) / L^{2}(x) \quad(x \rightarrow \infty)
$$

with a constant $C_{1}>0$ (cf. [16, Proposition 6.2, Eq(6.17)]). Recalling $m=\varepsilon(x \wedge|y|$ ) we find (3.27) to be true, provided that $\alpha \leq \beta<2 \alpha-1$.

Proof of $\left(\mathrm{b}^{*} 1\right)$ and $\left(\mathrm{c}^{*}\right)$. Instead of (3.15) one shows that if $\beta>\alpha$, for $m \leq M \lambda_{n}$ large enough,

$$
\sum_{k=1}^{n} \sum_{z<-m} p_{\{0\}}^{k-1}(x, w) p(z-w) p_{\{0\}}^{n-k}(z, y) \leq C m^{\alpha} F(-m)\left\{a(y) D_{n}(x)+a(-x) D_{n}(-y)\right\}
$$

the proof being the same as before except for a minor modification. ( $\left.b^{*} 1\right)$ readily follows from (3.28). As for $\left(c^{*}\right)$ we have the same result as in $\mathbf{P 4}$ but with $C^{+}=\infty$ instead of $E\left[|x|^{3} ; X<0\right]=\infty$ as well as with $\lambda_{n}$ replacing $\sqrt{n}$ (cf. [15, Proposition 2.1]) and combining it with (3.28) leads to the assertion as before.

Proof of $\left(\mathrm{b}^{*} 2\right)$. Using the bound $p_{\{0\}}^{n}(z, y) \leq C D_{n}(-y) a\left(-\lambda_{n}\right)$ valid for $z<-\lambda_{n}$ (see the dual of (3.25)) one can proceed as before.

\section{Proofs of Theorems}

Let $X^{(N)}$ be as in (2.7) and denote by $P_{(c, N)}^{*}$ the conditional probability law of the walk $S$, and hence of $X^{(N)}$, given the event (2.8):

$$
P_{(c, N)}^{*}[\cdots]=P\left[\cdots \mid S_{\lfloor N T\rfloor}^{b_{N}}=-c_{N}, \sigma_{A}^{b_{N}}>N T\right] .
$$


Let $\zeta=\zeta_{N}$ and $\zeta^{\prime}=\zeta_{N}^{\prime}$ stand for the first time $S^{b_{N}}$ enters $(-\infty, 0]$ and the last time $\leq N T$ it leaves $[1, \infty)$, respectively:

$$
\zeta=\sigma_{(-\infty, 0]}^{b_{N}} \quad \text { and } \quad \zeta^{\prime}=\max \left\{n \leq N T: S_{n}^{b_{N}} \in[1, \infty)\right\}
$$

The proofs of Theorems 1 and 2 are given separately according to whether $E\left[\left|S_{1}\right|^{3} ; S_{1}<0\right]$ is finite or infinity. We continue to use the notation of the preceding section. For simplicity we shall suppose $b_{N}=\left\lfloor b \sqrt{\sigma^{2} N}\right\rfloor$ and $c_{N}=\left\lfloor-c \sqrt{\sigma^{2} N}\right\rfloor$.

\subsection{Case $E\left[\left|S_{1}\right|^{3} ; S_{1}<0\right]<\infty$}

Under the conditional law $P_{(c, N)}^{*}$ once the walk $S^{b_{N}}$ goes down below the level $-\varepsilon \sqrt{N}$ with $\varepsilon>0$ prescribed, it never takes the positive value up to the time it terminates at $-c_{N}$ with the probability that approaches unity as $N \rightarrow \infty$ as we shall see (the second half of Lemma 4.1). On taking this for granted the convergence of the finite dimensional distribution of $X^{(N)}$ under $P_{(c, N)}^{*}$ follows immediately from P1(b) and P2(b): Noting $p_{A}^{n}(x, y) \sim p_{\{0\}}(x, y)$ as $|x| \wedge|y| \rightarrow \infty$ by $\mathbf{P 0}$, we infer that given $\eta_{k}<0<\xi_{j}\left(j=0, \ldots, m, k=0, \ldots, m^{\prime}\right)$ with $\xi_{0}=b$ and $\eta_{m^{\prime}}=-c$ and $0=s_{0}<s_{1}<\cdots<s_{m}<t_{0}<\cdots<t_{m^{\prime}}:=T$, we let $x_{j}=\left\lfloor\xi_{j} \sigma \sqrt{N}\right\rfloor, y_{k}=\left\lfloor\eta_{k} \sigma \sqrt{N}\right\rfloor, n_{j}=\left\lfloor N s_{j}\right\rfloor$ and $n_{k}^{\prime}=\left\lfloor N t_{k}\right\rfloor$. Then, as $N \rightarrow \infty$

$$
\begin{aligned}
& \left(\sqrt{\sigma^{2} N}\right)^{m+m^{\prime}} P_{(c, N)}^{*}\left[S_{n_{j}}^{b_{N}}=x_{j} \text { for } j \geq 1 \text { and } S_{n_{k}}^{b_{N}}=y_{k} \text { for } k \leq m^{\prime}-1\right] \\
& =\frac{\left(\sqrt{\sigma^{2} N}\right)^{m+m^{\prime}}}{p_{A}^{m+m^{\prime}}\left(x_{0}, y_{m^{\prime}}\right)} \prod_{j=1}^{m} p_{A}^{n_{j}-n_{j-1}}\left(x_{j-1}, x_{j}\right) \cdot p_{A}^{n_{0}^{\prime}-n_{m}}\left(x_{m}, y_{0}\right) \prod_{k=1}^{m^{\prime}} p_{A}^{n_{k}^{\prime}-n_{k-1}^{\prime}}\left(y_{k-1}, y_{k}\right) \\
& \longrightarrow \frac{\prod_{j=1}^{m} \mathfrak{g}_{s_{j}-s_{j-1}}^{0}\left(\xi_{j-1}, \xi_{j}\right) \cdot \rho_{t_{0}-s_{m}}\left(\xi_{m}-\eta_{0}\right) \prod_{k=1}^{m^{\prime}} \mathfrak{g}_{t_{k}-t_{k-1}}^{0}\left(\eta_{k-1}, \eta_{k}\right)}{\rho_{T}(b+c)}
\end{aligned}
$$

of which the right side is the density of the corresponding finite dimensional distribution of the limit process $X^{b, c, T}$ given by (2.2) - with apparent change of letters $x, y$ to $\xi, \eta$.

We need to show the tightness of the law of $X^{(N)}$ under $P_{(c, N)}^{*}$, or what amounts to the same thing [5], that for any $\varepsilon>0$ there exist $\delta>0$ and $N_{0}$ such that if $N \geq N_{0}$, then

$$
P_{(c, N)}^{*}\left[\sup _{0 \leq s<t \leq T} \sup _{|t-s|<\delta}\left|X_{t}^{(N)}-X_{s}^{(N)}\right|>\varepsilon\right]<\varepsilon .
$$

For $0 \leq j<k \leq N T$ let $\Lambda_{j, k}(\delta)$ denote the random variable

$$
\Lambda_{j, k}(\delta)=\sup _{\frac{1}{N} j \leq s<t \leq \frac{1}{N} k ;|t-s|<\delta}\left|X_{t}^{(N)}-X_{s}^{(N)}\right| .
$$

It suffices to show, instead of (4.3), that

$$
P_{(c, N)}^{*}\left[\Lambda_{0, \zeta}(\delta)>\varepsilon\right] \vee P_{(c, N)}^{*}\left[\Lambda_{\zeta, \zeta^{\prime}}(\delta)>2 \varepsilon, \zeta<\zeta^{\prime}\right] \vee P_{(c, N)}^{*}\left[\Lambda_{\zeta^{\prime}, N T}(\delta)>\varepsilon\right]<\varepsilon
$$

For simplicity we let $T=1$. First we show that

$$
P_{(c, N)}^{*}\left[\Lambda_{0, \zeta}(\delta)>\varepsilon\right]=P_{(c, N)}^{*}\left[\sup _{0 \leq s<t \leq \zeta ;|t-s|<\delta}\left|X_{t}^{(N)}-X_{s}^{(N)}\right|>\varepsilon\right]<\varepsilon .
$$


The conditional probability on the left side is expressed as

$$
P_{(c, N)}^{*}\left[\Lambda_{0, \zeta}(\delta)>\varepsilon\right]=\sum_{k=1}^{N} \sum_{z=1}^{\infty} P\left[\Lambda_{0, \zeta}(\delta)>\varepsilon, \zeta=k, S_{k}^{b_{N}}=-z\right] \frac{p_{A}^{N-k}\left(-z,-c_{N}\right)}{p_{A}^{N}\left(b_{N},-c_{N}\right)} .
$$

Splitting the inner summation at $M$, by $\mathbf{P} 4$ we can find $M>1$ such that the contribution to the double sum from $z>M$ is less than $\varepsilon / 2$, whereas by $\mathbf{P} \mathbf{1}(\mathrm{c}, \mathrm{d})$ it follows that for any $M>1$,

$$
\max _{k \leq N} \max _{1 \leq z \leq M} p_{A}^{N-k}\left(-z,-c_{N}\right) \leq C M / N .
$$

Since $p_{A}^{N}\left(b_{N},-c_{N}\right) \geq C^{\prime} / N$ according to $\mathbf{P} 2$, the latter bound shows that the ratio under the double summation sign is bounded by a constant $C_{M}$ for $z \leq M$, which together with the former one leads to

$$
P_{(c, N)}^{*}\left[\Lambda_{0, \zeta}(\delta)>\varepsilon\right] \leq C_{M} P\left[\Lambda_{0, \zeta}(\delta)>\varepsilon\right]+\varepsilon / 2,
$$

hence by the invariance principle, which entails that $P\left[\Lambda_{0, \zeta}(\delta)>\varepsilon\right]<\varepsilon / 2 C_{M}$ for $\delta>0$ small enough, we obtain (4.4).

The bound $P_{(c, N)}^{*}\left[\Lambda_{\zeta^{\prime}, N}(\delta)>\varepsilon\right]<\varepsilon$ follows from (4.4) as the dual assertion of it.

It remains to dispose of $P_{(c, N)}^{*}\left[\Lambda_{\zeta, \zeta^{\prime}}(\delta), \zeta<\zeta^{\prime}\right]$. Since $\Lambda_{\zeta, \zeta^{\prime}}(\delta)$ can not exceed $2 \varepsilon$ if the path of the walk is confined in the interval $[-\varepsilon \sqrt{N}, \varepsilon \sqrt{N}]$ for the time duration between $\zeta$ and $\zeta^{\prime}$, it suffices to show $\lim _{N \rightarrow \infty} P_{(c, N)}^{*}\left[\left|S_{k}^{b_{N}}\right| \leq \varepsilon \sqrt{N}\right.$ for $\left.k: \zeta \leq k \leq \zeta^{\prime}\right]=1$, which follows from the next lemma.

Lemma 4.1. If $E\left[\left|S_{1}\right|^{3} ; S_{1}<\infty\right]<\infty$, then there exists a constant $C$ depending on $b, c, A$ and $F$ such that for $R>1$

$$
P_{(c, N)}^{*}\left[S_{k}^{b_{N}}>R \text { for some } \zeta \leq k<N\right] \leq C / R
$$

and $P_{(c, N)}^{*}\left[S_{k}^{b_{N}}<-R\right.$ for some $\left.k<\zeta^{\prime}\right] \leq C / R$.

Proof. We have only to verify (4.5), the other relation being its dual. Let $E\left[\left|S_{1}\right|^{3} ; S_{1}<\infty\right]<$ $\infty$. Then $p_{A}^{N}\left(b_{N},-c_{N}\right) \sim C_{A, b, c} / N$ according to $\mathbf{P 2}(\mathrm{b})$, so that the conditional probability in (4.5) is dominated by a constant multiple of

$$
N \sum_{k=1}^{N} \sum_{z<0} h_{b_{N}}(k, z) P\left[\sigma_{[R, \infty)}^{z}<N-k<\sigma_{\{0\}}^{z}, S_{N-k}^{z}=-c_{N}\right],
$$

which is obviously less than

$$
N \sup _{x \geq R, 0<k<N} p_{\{0\}}^{N-k}\left(x,-c_{N}\right) \sum_{z<0} H_{(-\infty, 0]}^{b_{N}}(z) P\left[\sigma_{[R, \infty)}^{z}<\sigma_{\{0\}}^{z}\right] .
$$

We know that $P\left[\sigma_{[R, \infty)}^{z}<\sigma_{\{0\}}^{z}\right] \sim P\left[\sigma_{\{R\}}^{z}<\sigma_{\{0\}}^{z}\right]$ as $R \rightarrow \infty$ uniformly in $z \in \mathbb{Z}$ whenever $\lim _{x \rightarrow \infty} a(z) / a(-x)=0$ (cf. [16, Proposition 5.2(i)]), while

$$
P\left[\sigma_{\{R\}}^{z}<\sigma_{\{0\}}^{z}\right]=g_{\{0\}}(z, R) / g_{\{0\}}(R, R) \leq C^{\prime} a(z) / R,
$$


so that by (3.2) the sum in (4.6) is $O(1 / R), \lambda\left(b_{N}\right)$ being bounded under the present moment condition. Since the supremum in (4.6) is dominated by a constant multiple of $1 / c_{N}^{2} \sim$ $1 / c^{2} \sigma^{2} N$ owing to Lemma 3.1, we can conclude the asserted bound.

In the next subsection we shall need a tightness result with the conditional of avoiding $(-\infty, 0]$ instead of $A$. The following result, of which the condition $E\left[\left|S_{1}\right|^{3} ; S_{1}<\infty\right]<\infty$ is irrelevant, actually shows that the random walk bridge conditioned to stay positive weakly converges to a standard Brownian meander of length 1 pinned at a prescribed point $b$ at time 1 locally uniformly in $b>0$.

Lemma 4.2. For each $0<\varepsilon<1 / 2$, as $N \rightarrow \infty$ and $\delta \downarrow 0$ independently

$$
P\left[\Lambda_{0, n}(\delta)>\varepsilon \mid S_{n}^{b_{N}}=x, n<\zeta\right] \rightarrow 0
$$

uniformly for $\varepsilon N<n<N, 0<x<\varepsilon^{-1} \sqrt{N}$ and $\varepsilon<b<\varepsilon^{-1}$.

Proof. The main part of the proof will be given by means of the dual walk, denoted by $\hat{S}$ (defined by $\hat{S}_{k}^{x}=-S_{k}+x$ ). If $x \geq \eta \sqrt{N}$ for some constant $\eta>0$ the assertion is easy to show, since then $P\left[S_{n}^{b_{N}}=x, n<\zeta\right] \asymp P\left[S_{n}^{b_{N}}=x\right] \asymp 1 / \sqrt{N}$ (for $x, n$ subject to the condition of the lemma) according to $\mathbf{P 1}$ (b) and the problem is the same for the bridge without killing. For the proof of the lemma it therefore suffices to show that if $\eta<\frac{1}{2} \sigma \varepsilon$ and $\tau(\eta)$ is the last time $\leq n$ when $S^{b_{N}}$ leaves $[\eta \sqrt{N}, \infty)$, then

$$
\sup _{0<x<\eta \sqrt{N}} P\left[\Lambda_{0, \tau(\eta)+1}(\delta)>\frac{1}{2} \varepsilon \mid S_{n}^{b_{N}}=x, n<\zeta\right] \rightarrow 0,
$$

(as $N \rightarrow \infty$ and $\delta \rightarrow 0$ ) since $\Lambda_{\tau(\eta)+1, n}(\delta) \leq \eta / \sigma<\frac{1}{2} \varepsilon$. In order to separate the increment $S_{\tau(\eta)+1}-S_{\tau(\eta)}$ from $\Lambda_{0, \tau(\eta)+1}(\delta)$ we use the inequality

$$
\Lambda_{0, \tau(\eta)+1}(\delta) \leq \Lambda_{0, \tau(\eta)}(\delta)+\frac{1}{\sigma \sqrt{N}}\left|S_{\tau(\eta)}^{b_{N}}-S_{\tau(\eta)+1}^{b_{N}}\right| .
$$

Now we switch the description to that by the dual walk. Write $\tau_{*}^{x}(\eta)$ for $\hat{\sigma}_{[\eta \sqrt{N}, \infty)}^{x}$ where $\hat{\sigma}^{x}$ denotes the hitting time for $\hat{S}^{x}$. For any $\varepsilon^{\prime}>0$ we can choose $\eta<\frac{1}{2} \sigma \varepsilon$ and $N_{0}>1$ so that

$$
\sup _{0<x<\eta \sqrt{N}} P\left[\tau_{*}^{x}(\eta)>n / 2 \mid \hat{S}_{n}^{x}=b_{N}, n<\hat{\sigma}_{(-\infty, 0]}^{x}\right]<\varepsilon^{\prime} \quad \text { for } N>N_{0},
$$

for, by $\mathbf{P 0}$ and $\mathbf{P 1}$ (a), the conditional probability above is dominated by

$$
\frac{1}{p_{(-\infty, 0]}^{n}\left(x, b_{N}\right)} \sum_{z=1}^{\eta \sqrt{N}} p_{\{0\}}^{n / 2}(x, z) p_{\{0\}}^{n / 2}\left(z, b_{N}\right)<C \frac{\eta^{2} N x}{(n / 2)^{3 / 2}} \cdot \frac{\eta \sqrt{N} b_{N}}{(n / 2)^{3 / 2}} / \frac{x b_{N}}{N^{3 / 2}}<C_{\varepsilon} \eta^{3} .
$$

With the help of strong Markov property of $\hat{S}^{x}$ one applies what is mentioned above for the case $x \geq \eta \sqrt{N}$ to the walk $\hat{S}^{x}$ to find $\delta>0$ so that

$$
\sup _{0<x<\eta \sqrt{N}} P\left[\Lambda_{0, \tau_{*}^{x}(\eta)}(\delta)>\frac{1}{4} \varepsilon \mid S_{n}^{b_{N}}=x, n<\zeta\right]<\varepsilon^{\prime} .
$$

Since in this bound as well as in (4.8) $\varepsilon^{\prime}$ may be made arbitrarily small, (4.7) follows if we can show that

$$
P\left[\frac{1}{\sigma \sqrt{N}}\left(\hat{S}_{\tau_{*}^{x}(\eta)}^{x}-\hat{S}_{\tau_{*}^{x}(\eta)-1}^{x}\right)>\frac{1}{4} \varepsilon, \tau_{*}^{x}(\eta) \leq \frac{1}{2} n \mid \hat{S}_{n}^{x}=b_{N}, n<\hat{\sigma}_{(-\infty, 0]}^{x}\right] \rightarrow 0
$$


for any prescribed $\eta>0$.

Our proof of (4.9) rests on the following facts: for $0<x<\eta \sqrt{N}$,

$$
\begin{aligned}
& P\left[\hat{S}_{\tau_{*}^{x}(\eta)}^{x}-\eta \sqrt{N}>\eta \sqrt{N}, \tau_{*}^{x}(\eta)<\hat{\sigma}_{(-\infty, 0]}^{x}\right]=(x / \eta \sqrt{N}) \times o(1), \\
& p_{(-\infty, 0]}^{n}\left(x, b_{N}\right) \geq c_{\varepsilon} x / n \quad(\varepsilon N<n<N) .
\end{aligned}
$$

(In (b) $c_{\varepsilon}$ is a positive constant that may depend on $\varepsilon$.) Here (a) follows from P5 and (b) from P0. Let $\mathcal{E}$ stand for the event

$$
\mathcal{E}:=\left\{\frac{1}{\sigma \sqrt{N}}\left(\hat{S}_{\tau_{*}^{x}(\eta)}^{x}-\hat{S}_{\tau_{*}^{x}(\eta)-1}^{x}\right)>\frac{1}{4} \varepsilon, \tau_{*}^{x}(\eta) \leq \frac{1}{2} n \wedge \hat{\sigma}_{(-\infty, 0]}^{x}\right\} .
$$

For simplicity we suppose $n$ to be even. Applying (4.10 $\mathrm{b}$ ) to the dual walk with the help of the trivial bound $p_{(-\infty, 0]}^{\lfloor n / 2\rfloor}\left(z, b_{N}\right) \leq C / \sqrt{n}$ leads to

$$
P\left[\hat{S}_{n / 2}^{z}=b_{N}, n / 2<\hat{\sigma}_{(-\infty, 0]}^{z}\right] \leq C^{\prime}(\sqrt{n} / x) P\left[\hat{S}_{n}^{x}=b_{N}, n<\hat{\sigma}_{(-\infty, 0]}^{x}\right] \quad(z>0)
$$

and hence

$$
\begin{aligned}
P\left[\mathcal{E}, \hat{S}_{n}^{x}=b_{N}, n<\hat{\sigma}_{(-\infty, 0]}^{x}\right] & =\sum_{z=1}^{\infty} P\left[\mathcal{E}, \hat{S}_{n / 2}^{x}=z\right] P\left[\hat{S}_{n / 2}^{z}=b_{N}, n / 2<\hat{\sigma}_{(-\infty, 0]}^{z}\right] \\
& \leq C^{\prime}(\sqrt{n} / x) P(\mathcal{E}) P\left[\hat{S}_{n}^{x}=b_{N}, n<\hat{\sigma}_{(-\infty, 0]}^{x}\right] .
\end{aligned}
$$

The constant $\eta$ may be supposed to be smaller than $\frac{1}{8} \sigma \varepsilon$ so that the occurrence of $\mathcal{E}$ entails that $\hat{S}_{\tau_{*}^{x}(\eta)}^{x}>\frac{1}{4} \sigma \varepsilon \sqrt{N}>2 \eta \sqrt{N}$, and hence that

$$
\mathcal{E} \subset\left\{\hat{S}_{\tau_{*}^{x}(\eta)}^{x}-\eta \sqrt{N}>\eta \sqrt{N}, \tau_{*}^{x}(\eta) \leq \hat{\sigma}_{(-\infty, 0]}^{x}\right\},
$$

hence $P(\mathcal{E})=[x / \eta \sqrt{N}] \times o(1)$ according to (4.10a), and we may conclude

$$
P\left[\mathcal{E}, \hat{S}_{n}^{x}=b_{N}, n<\hat{\sigma}_{(-\infty, 0]}^{x}\right]=P\left[\hat{S}_{n}^{x}=b_{N}, n<\hat{\sigma}_{(-\infty, 0]}^{x}\right] \times o(1),
$$

which is the same as (4.9). Proof of Lemma 4.2 is finished.

\subsection{Case $E\left[\left|S_{1}\right|^{3} ; S_{1}<0\right]=\infty$}

Here we suppose in addition to $E\left[\left|S_{1}\right|^{3} ; S_{1}\right]=\infty$ that $F(-u)$ is regularly varying as $u \rightarrow \infty$ with index $-\beta \in[-3,-2]$.

Given $M>1$ we shall let $x \wedge(-y) \wedge n \rightarrow \infty$ under the constraint

$$
M^{-1} \leq x / \sqrt{n} \leq M \quad \text { and } \quad M^{-1} \leq-y / \sqrt{n} \leq M .
$$

The following result refines the estimates given in $\mathbf{P 3}$ and $\mathbf{P} 4$ under the present assumption.

Proposition 4.1. As $x \wedge(-y) \wedge n \rightarrow \infty$ under 4.12)

(i) if $2 \leq \beta<3$,

$$
p_{A}^{n}(x, y) \sim \sum_{k=1}^{n} \sum_{z=1}^{\infty} \sum_{w=1}^{\infty} \mathfrak{g}_{\sigma^{2} k}^{0}(x, z) \frac{\beta F(-w-z)}{w+z} \mathfrak{g}_{\sigma^{2}(n-k)}^{0}(-w, y) ;
$$

(ii) if $\beta=3$ and $E\left[\left|S_{1}\right|^{3} ; S_{1}<0\right]=\infty$,

$$
p_{A}^{n}(x, y) \sim \rho_{\sigma^{2} n}(x+|y|) \frac{2}{\sigma^{2}} \int_{0}^{\sqrt{n}} F(-u) u^{2} d u .
$$


We write

$$
\xi_{N}=x / \sqrt{\sigma^{2} N} \text { and } \eta_{N}=y / \sqrt{\sigma^{2} N} .
$$

Recall the definition of $J_{t}(\xi, \eta)$. An elementary computation derives from Proposition 4.1 the following

Corollary 4.1. For any $M>1$ uniformly for $x, y, n$ satisfying (4.12) and $M^{-1}<n / N<M$, as $N \rightarrow \infty$,

$$
p_{A}^{n}(x, y) \sim \begin{cases}\sigma^{-\beta-1}[\sqrt{N} F(-\sqrt{N})] J_{n / N}\left(\xi_{N}, \eta_{N}\right) & \beta<3, \\ \sigma^{-4}\left[2 \int_{0}^{\sqrt{N}} F(-u) u^{2} d u / N\right] \rho_{n / N}\left(\xi_{N}+\eta_{N}\right) & \beta=3 .\end{cases}
$$

The convergence of finite dimensional distributions of $X^{N}$ — given by a formula analogous to (4.2) - follows from Corollary 4.1. The proof of Theorems 1 and 2 is accordingly accomplished if we verify Proposition 4.1 as well as the tightness of the conditional law of $X^{(N)}$. The verification of Proposition 4.1 is given in the paragraphs 4.2.1 and 4.2.2 for the cases $\beta<3$ and $\beta=3$ respectively. The tightness proof is given in the paragraph 4.2.3.

\subsubsection{Proof of (i) of Proposition 4.1.}

Let $\beta<3$ and $\zeta_{x}$ and $\zeta_{x}^{\prime}$ be defined as in (4.1) with $x$ in place of $b_{N}$. We first observe that as $x \wedge(-y) \wedge n \rightarrow \infty$ under (4.12) and $\varepsilon \downarrow 0$ (independently)

$$
P\left[S_{\zeta_{x}-1}^{x} \wedge\left(-S_{\zeta_{x}}^{x}\right)>\varepsilon \sqrt{n} \text { and } \zeta_{x}^{\prime}=\zeta_{x}-1 \mid S_{n}^{x}=y, \sigma_{A}^{x}>n\right] \rightarrow 1
$$

and

$$
P\left[\varepsilon n<\zeta_{x}<(1-\varepsilon) n \mid S_{n}^{x}=y, \sigma_{A}^{x}>n\right] \rightarrow 1 .
$$

(44.14) is proved also in case $\beta=3$ but by a different approach in the next paragraph 4.2.2.) (4.13) follows immediately from Corollary 3.1(a,b). For the proof of (4.14), recalling the convention max $A=0$ consider the representation

$$
p_{A}^{n}(x, y)=\sum_{k=1}^{n} \sum_{z=1}^{\infty} \sum_{z^{\prime} \in(-\infty, 0] \backslash A} p_{(-\infty, 0]}^{k-1}(x, z) p\left(z^{\prime}-z\right) p_{A}^{n-k}\left(z^{\prime}, y\right) .
$$

It then suffices to show that for any $\eta>0$ there exists $\varepsilon>0$ such that the sum in (4.15) restricted to $k \leq \varepsilon n$ is at most $\eta$, the part $k>(1-\varepsilon) n$ being disposed of by duality because of (4.13). We may restrict the summation over $z$ and $z^{\prime}$ to $z \wedge\left(-z^{\prime}\right)>\theta \sqrt{n}$ with a positive constant $\theta$ because of (4.13) again . The proof is therefore finished if we show that for any $\eta>0$ and $\theta>0$ there exists $\varepsilon>0$ such that

$$
\sum_{k \leq \varepsilon n} \sum_{z>\theta \sqrt{n}} \sum_{w>\theta \sqrt{n}} \frac{P\left[S_{k-1}^{x}=z\right] p(-w-z) p_{A}^{n-k}(-w, y)}{p_{A}^{n}(x, y)} \leq \eta .
$$

On using the trivial bound $p_{A}^{n-k}(-w, y) \leq C / \sqrt{n-k}$ and $\mathbf{P 3}$ in turn this triple sum is dominated by

$$
\frac{C}{\sqrt{n}} \sum_{k \leq \varepsilon n} \frac{F(-2 \theta \sqrt{n})}{p_{A}^{n}(x, y)} \leq C^{\prime} \theta^{-\beta} \frac{\varepsilon n^{1 / 2} F(-\sqrt{n})}{p_{A}^{n}(x, y)} \leq \varepsilon C_{M} \frac{\theta^{-\beta} n^{3 / 2} F(-\sqrt{n})}{1+\sum_{w=1}^{\sqrt{n}} w^{3} p(-w)} .
$$


Since the last ratio is bounded, the right-most member becomes arbitrarily small along with $\varepsilon$, as desired. Thus (4.14) has been proved.

In view of P0 $p_{(-\infty, 0]}^{k}$ and $p_{A}^{k}$ being interchangeable, we infer from (4.13) and (4.14) that

$$
p_{A}^{n}(x, y)=\sum_{k=\lfloor\varepsilon n\rfloor}^{\lfloor(1-\varepsilon) n\rfloor} \sum_{z \geq \varepsilon \sqrt{n}} \sum_{w \geq \varepsilon \sqrt{n}} p_{A}^{k}(x, z) p(-w-z) p_{A}^{n-k}(-w, y)\left\{1+o_{\varepsilon}(1)\right\},
$$

where $o_{\varepsilon}(1) \rightarrow 0$ as $n \rightarrow \infty$ under (4.12) and $\varepsilon \downarrow 0$. In this triple sum we may replace $p_{A}^{n-k}(-w, y)$ by $\mathfrak{g}_{\sigma^{2}(n-k)}^{0}(-w, y)$ owing to $\mathbf{P} \mathbf{1}$ since the contribution to the sum from $w>$ $M^{\prime} \sqrt{n}$ becomes negligible as $M^{\prime} \rightarrow \infty$ as is assured without difficulty. Similarly $p_{A}^{k}(x, z)$ may be replaced by $\mathfrak{g}_{\sigma^{2} k}^{0}(x, z)$. Since either of the sums over $0<w<\varepsilon \sqrt{n}$ and $0<z<\varepsilon \sqrt{n}$ contributes only $o_{\varepsilon}(1)$ and for reason of symmetry Proposition 4.1 (i) therefore follows from the next lemma.

Lemma 4.3. Suppose that $F(-t)=t^{-\beta} L_{-}(t), t \geq 1$ with a slowly varying function $L_{-}$and $\beta>1$. Then for each $M>1$, as $z \wedge(-y) \wedge n \rightarrow \infty$ under $M^{-1} \leq-y / \sqrt{n} \leq M$ and $z \leq M \sqrt{n}$

$$
\sum_{w=1}^{\infty} p(-w-z) \mathfrak{g}_{n}^{0}(-w, y) \sim \sum_{w=1}^{\infty} \frac{\beta L_{-}(w+z)}{(w+z)^{\beta+1}} \mathfrak{g}_{n}^{0}(-w, y)
$$

Proof. On summing by parts,

$$
\sum_{w=1}^{\infty} p(-w-z) \mathfrak{g}_{n}^{0}(-w, y)=\sum_{w=1}^{\infty} F(-w-z)\left[-\mathfrak{g}_{n}^{0}(-w+1, y)+\mathfrak{g}_{n}^{0}(-w, y)\right] .
$$

We can choose a slowly varying function $\tilde{L}$ so that $L_{-}(t)=\tilde{L}_{-}(t)(1+\delta(t))$ with $\tilde{L}_{-}^{\prime}(t)=$ $o\left(L_{-}(t) / t\right)$ and $\delta(t) \rightarrow 0$ so that $\left[t^{-\beta} \tilde{L}_{-}(t)\right]^{\prime}=\left(\beta t^{-\beta-1}\right) L_{-}(t)(-1+o(1))$ as $t \rightarrow \infty$. Then substituting $F(-w-z)=(w+z)^{-\beta} \tilde{L}_{-}(w+z)(1+\delta(w+z))$, and summing by parts back, the sum of the last series above is written as

$$
\sum_{w=1}^{\infty} \beta(w+z)^{-\beta-1} L_{-}(w+z) \mathfrak{g}_{n}^{0}(-w, y)\{1+o(1)\}+r(z, y, n),
$$

where $r(z, y, n)=\sum_{w=1}^{\infty}\left[\mathfrak{g}_{n}^{0}(-w+1, y)-\mathfrak{g}_{n}^{0}(-w, y)\right](w+z)^{-\beta} \tilde{L}_{-}(w+z) \delta(w+z)$. Noting

$$
\left|\mathfrak{g}_{n}^{0}(-w+1, y)-\mathfrak{g}_{n}^{0}(-w, y)\right|<C / n \text { for all } w, y,
$$

we deduce that

$$
|r(z, y, n)| \leq \frac{C}{n} \sum_{j \geq z} j^{-\beta} \tilde{L}_{-}(j) \delta(j)=\frac{L_{-}(z)}{n z^{\beta-1}} \times o(1) .
$$

On the other hand if $-y \asymp \sqrt{n}$ and $1<z=O(\sqrt{n})$, then the sum on the right side of (4.16) is bounded from below by a positive multiple of

$$
\sum_{z \wedge \sqrt{n} \leq w \leq 2(z \wedge \sqrt{n})} \frac{L_{-}(w+z) w|y|}{(w+z)^{\beta+1} n^{3 / 2}} \geq C^{\prime} \frac{L_{-}(z)}{n z^{\beta-1}}
$$

with $C^{\prime}$ a positive constant, showing $r(z, y, n)$ is negligible. This finishes the proof. 


\subsubsection{Proof of (ii) of Proposition 4.1.}

Let $\beta=3$ so that $F(-u)=u^{-3} L_{-}(u), u>0$ with a slowly varying $L_{-}$, and put

$$
L_{-}^{*}(u)=\int_{1}^{u} \frac{L_{-}(s)}{s} d s,
$$

which is also slowly varying. Recall $L_{-}^{*}(u) / L_{-}(u) \rightarrow \infty$ as $u \rightarrow \infty$, the fact that differentiates the case $\beta=3$ from the case $\beta<3$ (as is exhibited by Lemma 3.2).

We follow the proof of P2 given in [13, pp.702-703] for the special case $A=\{0\}$. As therein, take a small $\varepsilon>0$ and break the sum in the expression of $p_{A}^{n}(x, y)$ given in (4.15) into three parts by splitting the range of $k$ according as $1 \leq k<\varepsilon n$, $\varepsilon n \leq k<(1-\varepsilon) n$ and $(1-\varepsilon) n \leq k \leq n$, and call the corresponding sum $I, I I$ and $I I I$.

On using the second half of $\mathbf{P 6}$ and the bound $p_{A}^{n-k}(w, y) \leq C(|w y| \wedge n) / n^{3 / 2}(k<\varepsilon n)$ the part $I$ is dominated by a constant multiple of

$$
\sum_{k \leq \varepsilon n}\left(\frac{1}{\sqrt{k} x} \sum_{0<w<\sqrt{n}} H_{(-\infty, 0]}^{+\infty}(-w) \frac{|w y|}{n^{3 / 2}}+\frac{1}{\sqrt{n}} \sum_{w>\sqrt{n}} p_{A}^{k-1}(x,-w) F(-w)\right) .
$$

By the present assumption about $F$

$$
H_{(-\infty, 0]}^{+\infty}(-w) \sim \frac{2}{\sigma^{2}} \sum_{z>w} F(-z) \sim \frac{L_{-}(-w)}{\sigma^{2} w^{2}} \quad(w \rightarrow \infty)
$$

(cf. [14, (2.7)]). Under (4.12) $y / x \leq M^{2}$ and easy computations deduce that

$$
I \leq C_{M}\left[\sqrt{\varepsilon} L_{-}^{*}(\sqrt{n}) / n+\varepsilon L_{-}(\sqrt{n}) / n\right]<C_{M}^{\prime} \sqrt{\varepsilon} L_{-}^{*}(\sqrt{n}) / n .
$$

III admits the same bound as a dual relation since $P_{(c, N)}^{*}\left[\zeta=\zeta^{\prime}+1\right] \rightarrow 1$ by Corollary 3.1 .

As for $I I$ we note that for arbitrarily small $\eta>0$ the range of the variable $w$ may be restricted to $w<\eta \sqrt{n}$ in view of Lemma $3.2(\mathrm{c})$. Then by the first half of $\mathbf{P} 6$ and $\mathbf{P} \mathbf{1}(\mathrm{d})$

$$
I I=\sum_{\varepsilon n \leq k \leq(1-\varepsilon) n} \frac{f_{+}(x) \mathfrak{g}_{\sigma^{2} k}(x)}{k} \sum_{0<w<\eta \sqrt{n}} H_{(-\infty, 0]}^{+\infty}(-w) p_{A}^{n-k}(-w, y)\left(1+o_{\varepsilon, \eta}(1)\right) .
$$

Here for each pair of $\varepsilon$ and $\eta, o_{\varepsilon, \eta}(1) \rightarrow 0$ as $n \rightarrow \infty$ uniformly for $x, y$ satisfying (4.12).

By $\mathbf{P} 1 p_{A}^{n-k}(-w, y) \sim 2\left[\sigma^{2}(n-k)\right]^{-1}|w y| \mathfrak{g}_{\sigma^{2}(n-k)}(y)$ as $w \rightarrow \infty$ under $w=o(\sqrt{n})$ and observe, on replacing $f_{+}(x)$ by $x$,

$$
I I=\sum_{\varepsilon n \leq k \leq(1-\varepsilon) n} \frac{2 x|y| \mathfrak{g}_{\sigma^{2} k}(x) \mathfrak{g}_{\sigma^{2}(n-k)}(y)}{\sigma^{2} k(n-k)} \sum_{0<w<\eta \sqrt{n}} H_{(-\infty, 0]}^{+\infty}(-w) w\left(1+o_{\varepsilon, \eta}(1)\right) .
$$

Writing $x_{n}=x / \sigma \sqrt{n}, y_{n}=y / \sigma \sqrt{n}$ we see $x \mathfrak{g}_{\sigma^{2} k}(x) / k=\rho_{k}(x / \sigma)=\rho_{k / n}\left(x_{n}\right) / n$ and

$$
\sum_{\varepsilon n \leq k \leq(1-\varepsilon) n} \frac{2 x|y| \mathfrak{g}_{\sigma^{2} k}(x) \mathfrak{g}_{\sigma^{2}(n-k)}(y)}{\sigma^{2} k(n-k)}=\frac{2}{n \sigma^{2}} \int_{0}^{1} \rho_{t}\left(x_{n}\right) \rho_{1-t}\left(\left|y_{n}\right|\right) d t+O\left(\frac{\varepsilon}{n}\right)+o\left(\frac{1}{n}\right) .
$$

Here we have used the condition (4.12) again. On the other hand in view of (4.18)

$$
\sum_{0<w<\eta \sqrt{n}} H_{(-\infty, 0]}^{+\infty}(-w) w\left(1+o_{\varepsilon, \eta}(1)\right)=\frac{1}{\sigma^{2}} L_{-}^{*}(\sqrt{n})\left(1+o_{\varepsilon}(1)\right) .
$$


Finally observing $\int_{0}^{1} \rho_{t}\left(x_{n}\right) \rho_{1-t}\left(\left|y_{n}\right|\right) d t=\rho_{1}\left(x_{n}+\left|y_{n}\right|\right)=\rho_{\sigma^{2} n}(x+|y|) \sigma^{2} n$ we find that

$$
I I=\frac{2}{\sigma^{2}}\left[\left(\rho_{\sigma^{2} n}(x+|y|)+O(\varepsilon / n)\right] L_{-}^{*}(\sqrt{n})\left(1+o_{\varepsilon}(1)\right),\right.
$$

which together with the bounds of $I$ and $I I I$ verifies the formula in (ii).

\subsubsection{Proof of tightness.}

It suffices to show that for any $\varepsilon$ there exists $N_{0}$ and $\delta>0$ such that

$$
P_{(c, N)}^{*}\left[\Lambda_{0, \zeta-1}(\delta)>\varepsilon\right]<\varepsilon \quad \text { for } \quad N \geq N_{0}
$$

$\left(\zeta\right.$ and $\zeta^{\prime}$ are given in (4.1) ), since $P_{(c, N)}^{*}\left[\zeta=\zeta^{\prime}+1\right] \rightarrow 1$ in view of Corollary 3.1(a). Observe that $P\left[\Lambda_{0, \zeta-1}(\delta)>\varepsilon, \zeta=k, S_{k}^{b_{N}}=-w\right], w \geq 1$ may be written as

$$
\sum_{x=1}^{\infty} P\left[\Lambda_{0, k-1}(\delta)>\varepsilon, k \leq \zeta, S_{k-1}^{b_{N}}=x\right] p(-w-x) .
$$

By the dual assertion of Lemma $3.2(\mathrm{~b}, \mathrm{c})$ one can choose $M$ so large that $P_{(c, N)}^{*}\left[S_{\zeta-1}^{b_{N}} \geq\right.$ $M \sqrt{N}]<\varepsilon / 2$ and accordingly obtains that $P_{(c, N)}^{*}\left[\Lambda_{0, \zeta-1}(\delta)>\varepsilon\right]$ is at most $\varepsilon / 2$ plus

$$
\sum_{k=1}^{N} \sum_{0<x<M \sqrt{N}} \sum_{w=1}^{\infty} \frac{P\left[\Lambda_{0, k-1}(\delta)>\varepsilon, k \leq \zeta, S_{k-1}^{b_{N}}=x\right] p(-w-x) p_{A}^{N-k}\left(-w,-c_{N}\right)}{p_{A}^{N}\left(b_{N},-c_{N}\right)}
$$

By Lemma 4.2, as $\delta \downarrow 0$ and $N \rightarrow \infty, P\left[\Lambda_{0, k-1}(\delta)>\varepsilon \mid k \leq \zeta, S_{k-1}^{b_{N}}=x\right] \rightarrow 0$ or, what amounts to the same,

$$
P\left[\Lambda_{0, k-1}(\delta)>\varepsilon, k \leq \zeta, S_{k-1}^{b_{N}}=x\right]=p_{(-\infty, 0]}^{k-1}\left(b_{N}, x\right) \times o(1)
$$

uniformly for $\eta N \leq k \leq(1-\eta) N$ and $0<x<M \sqrt{N}$ for each positive $\eta$. On recalling (4.15) this disposes of the contribution from $\eta N \leq k \leq(1-\eta) N$. It follows from (4.14) (if $\beta<3$ ) and (4.19) (if $\beta=3$ ) that the contribution from $k<\eta N$ is negligible; and similarly for that from $k>(1-\eta) N$, the proof of tightness is complete.

\subsection{Notes on the proofs of Theorems 3 and 4}

The proofs are similar to those of Theorems 1 and 2 and we do not present them but indicate some points that make difference from the latter. Throughout this section we assume (2.11) to be valid, put $b_{N}=\left\lfloor b \lambda_{n}\right\rfloor$ and $c_{N}=\left\lfloor c \lambda_{n}\right\rfloor$ and let $P_{(c, N)}^{*}, \zeta$ and $\zeta^{\prime}$ be defined as before. First we note that the propositions corresponding to $\mathbf{P} \mathbf{1}$ through $\mathbf{P} \mathbf{4}$ and $\mathbf{P} \mathbf{6}$ for the case $\sigma^{2}=\infty$ are obtained in [15]: specifically the corresponding results of [15] are

$$
\begin{aligned}
& \mathbf{P 1}(\mathrm{a}, \mathrm{b}) \mapsto \text { Theorem 3, Corollary 3, Proposition 2.1; } \mathbf{P 1}(\mathrm{c}) \mapsto \text { Lemma } 5.2[([3.21)] ; \\
& \mathbf{P 1}(\mathrm{d}) \mapsto \text { Proposition 2.3(ii) }[([3.26)] ; \quad \mathbf{P 2} \mapsto \text { Theorem } 4 ; \\
& \mathbf{P} \mathbf{3} \mapsto \text { Proposition } 6.1 ; \quad \mathbf{P} \mathbf{4} \mapsto \text { Proposition } 2.2 ; \quad \mathbf{P 6} \mapsto \text { Lemma } 6.5,
\end{aligned}
$$

where Theorems, Propositions or Lemmas are those of [15], of which a few are already used in Section 4, their reference numbers being indicated in the square brackets. The result corresponding to $\mathbf{P 0}$ (in particular its first one saying $p_{\{0\}}^{n}(x, y) \sim p_{(-\infty, 0]}^{n}(x, y)$ ) that is 
missing in the above list and used in the proofs of Corollary 3.1 and Lemma 4.2 does not hold in the relevant range $0<x, y \leq \lambda_{n}$ but is valid if restricted to $x, y \in\left[M^{-1} \lambda_{n}, M \lambda_{n}\right]$. Corollary 3.1 was used only under this restriction, while a result corresponding to Lemma 4.2 will be proved below. As for P5 also missing in the above, we need a corresponding one in a dual form, which will be presented below in (4.24).

It is shown in [15, Theorem 6] that as $N \rightarrow \infty$

$$
p_{A}^{N}\left(b^{N},-c^{N}\right) \sim C_{A}^{+}(b+c) \mathfrak{p}_{1}(b+c) / N \quad \text { if } \quad C^{+}<\infty
$$

where $C_{A}^{+}$is some positive constant. Instead of Lemmas 4.1 and 4.2 we obtain the following Lemmas 4.4 and 4.5, respectively.

Lemma 4.4. Suppose $C^{+}<\infty$. Then there exists a constant $C$ depending on $b, c, A$ and $F$ such that for $R>1$

$$
P_{(c, N)}^{*}\left[S_{k}^{b_{N}}>R \text { for some } \zeta \leq k \leq N\right] \leq C / a(-R)
$$

and $P_{(c, N)}^{*}\left[S_{k}^{b_{N}}<-R\right.$ for some $\left.k<\zeta^{\prime}\right] \leq C / a(-R)$.

Proof. As in the proof of Lemma 4.1 we see that $P\left[\sigma_{[R, \infty)}^{z}<\sigma_{\{0\}}^{z}\right] \leq C_{2} a(z) / a(-R)(z<0)$ and owing to Lemma $3.3 \sup _{x>R} p_{\{0\}}^{N-k}\left(x,-c_{N}\right) \leq C_{1} L\left(c_{N}\right) /\left[c_{N}\right]^{\alpha} \sim C_{1} / c^{\alpha} N(k<N)$ and that by (4.22) and (2.13) the probability in (4.23) is at most a constant multiple of

$$
\frac{1}{p_{A}^{N}\left(b^{N},-c^{N}\right)} \sum_{z=-\infty}^{-1} P\left[S_{\zeta}^{b_{N}}=z\right] \frac{a(z)}{N a(-R)} \sim C_{A, b, c} \frac{a\left(b_{N}\right)}{a(-R)} \leq \frac{C}{a(-R)},
$$

where we have also used relation (3.20) that gives $E\left[a\left(S_{\zeta}^{b_{N}}\right)\right]=a\left(b_{N}\right)$.

For $0 \leq j<k \leq N T$ let $\Lambda_{j, k}^{\prime \prime}(\delta)$ denote the random variable

$$
\Lambda_{j, k}^{\prime \prime}(\delta)=\sup _{\frac{1}{N} j \leq s<t \leq \frac{1}{N} k ;|t-s|<\delta} \sup _{s<u<t}\left|X_{u}^{(N)}-X_{s}^{(N)}\right| \wedge\left|X_{t}^{(N)}-X_{u}^{(N)}\right|
$$

Lemma 4.5. For each $0<\varepsilon<1 / 2$, as $N \rightarrow \infty$ and $\delta \downarrow 0$ independently

$$
P\left[\Lambda_{0, n}^{\prime \prime}(\delta)>\varepsilon \mid S_{n}^{b_{N}}=x, n<\zeta\right] \rightarrow 0
$$

uniformly for $\varepsilon N<n<N$ and $0<x<\varepsilon^{-1} \lambda_{N}$.

Proof. As in the proof of Lemma 4.2 the proof is reduced to verification of (4.9) modified in an obvious way (except for the existence of $\eta$ that makes (4.8) valid (with $\eta \sqrt{N}$ replaced by $\eta \lambda_{N}$ ) for which we use the convergence of the normalized walk conditioned to stay positive to a stable meander (as found in [7]). The verification of (4.9) is carried out in the same line if an appropriate substitute for (4.10) (and accordingly that for (4.11)) is given. Let $U_{\text {as }}$ denote the renewal function of the ascending ladder height process of the walk $\left(S_{n}\right)$. Then, as $R \rightarrow \infty$

$$
P\left[\hat{S}_{\sigma_{[R, \infty)}}^{x} \geq R+r, \hat{\sigma}_{[R, \infty)}<\hat{\sigma}_{(-\infty, 0]}\right]=\frac{U_{\mathrm{as}}(x)}{U_{\mathrm{as}}(R+r)-U_{\mathrm{as}}(R)} \times o(1)
$$


uniformly for $0<x<R, r \geq 1$ according to [17, Lemma 1.1]. From Proposition 11 of [8] we infer that for each $M>1$ there exists a constant $c_{\circ}>0$ such that

$$
\begin{aligned}
p_{(-\infty, 0]}^{n}(y, x) & =P\left[\hat{S}_{n}^{x}=y, n<\hat{\sigma}_{(-\infty, 0]}^{x}\right] \\
& \geq c_{\circ} U_{\text {as }}(x) P\left[\sigma_{[1, \infty)}^{0}>n\right] / \lambda_{n} \quad\left(0<x<M^{-1} \lambda_{n}<y<M \lambda_{n}\right) .
\end{aligned}
$$

Moreover $U_{\text {as }}$ varies regularly (with index $\alpha-1$ ) and $U_{\text {as }}\left(\lambda_{n}\right) P\left[\sigma_{[1, \infty)}^{0}>n\right]$ approaches a positive constant as $n \rightarrow \infty$. From these facts one deduces that for $0<x<\eta \lambda_{N}$,

(a) $P\left[\hat{S}_{\tau_{*}(\eta)}^{x}-\eta \lambda_{N}>\eta \lambda_{N}, \tau_{*}^{x}(\eta)<\hat{\sigma}_{(-\infty, 0]}^{x}\right]=\left[U_{\text {as }}(x) / U_{\text {as }}\left(\lambda_{N}\right)\right] \times o(1)$,

(b) $\quad P\left[\hat{S}_{n}^{x}=b_{N}, n<\hat{\sigma}_{(-\infty, 0]}^{x}\right] \geq c_{\varepsilon}\left[U_{\mathrm{as}}(x) / U_{\mathrm{as}}\left(\lambda_{N}\right)\right] / \lambda_{N} \quad(\varepsilon N<n<N)$

instead of (4.10), and then

$$
P\left[\hat{S}_{n / 2}^{z}=b_{N}, n / 2<\hat{\sigma}_{(-\infty, 0]}^{z}\right] \leq C\left[U_{\text {as }}\left(\lambda_{N}\right) / U_{\text {as }}(x)\right] P\left[\hat{S}_{n}^{x}=b_{N}, n<\hat{\sigma}_{(-\infty, 0]}^{x}\right] \quad(z>0)
$$

instead of (4.11). The rest of proof is easy and omitted.

With these two lemmas as well as those corresponding to $\mathbf{P} \mathbf{1}$ to $\mathbf{P} \mathbf{6}$ one can follow the arguments of Sections 4.1 and 4.2 to prove Theorems 3 and 4 .

\section{Appendix}

(A) Let $\ell(t)$ be the local time at the origin of the Brownian motion $W$. For a constant $\lambda>0$ let $\xi_{t}^{(\lambda)}, t \geq 0$ be $W$ killed at the rate $\lambda \ell(t)$; in other words, $\xi^{(\lambda)}$ is a Markov process whose sample path is continuous and whose transition probability is given by

$$
q_{t}^{(\lambda)}(\xi, \eta)=E_{\xi}\left[e^{-\lambda \ell(t)} ; W_{t} \in d \eta\right] / d \eta
$$

Here $P_{\xi}$ denotes the probability law of $W^{\xi}$ and $\xi$ being dropped from $W^{\xi}$ under $P_{\xi}$. Then the conditional law of the process $\xi^{(\lambda)}$ given $\xi_{0}^{(\lambda)}=b$ and $\xi_{T}^{(\lambda)}=-c$ converges weakly, as $\lambda \rightarrow \infty$, to the law of the process $X^{b, c, T}$ described in (2.2) and the density of finite dimensional distribution of the limit process given on the right side of (4.2) is expressed as

$$
\lim _{\lambda \rightarrow \infty} \frac{\lambda E_{b}\left[e^{-\lambda \ell(T)} ; \cap_{j=1}^{m}\left\{W_{t_{j}} \in d \xi_{j}\right\} \text { and } \cap_{k=0}^{m^{\prime}}\left\{W_{t_{j}} \in d \eta_{j}\right\}\right]}{\rho_{T}(b+c) d \xi_{1} \cdots d \xi_{m} \cdot d \eta_{0} \cdots d \eta_{m^{\prime}}}
$$

We prove only the latter half of the assertion, the other half being argued similarly to what is done in Section 4.1 for the random walk. The proof rests on the formula

$$
P_{0}\left[\ell(t) \in d u, W_{t} \in d \eta\right]=\rho_{t}(|\eta|+u) d u d \eta
$$

(cf. [9, Problem 2.2.3], [11, Eq(VI.2.18)] etc.), from which we derive

(i) $\lim _{\lambda \rightarrow \infty} q_{t}^{(\lambda)}(\xi, \eta)=\mathfrak{g}_{t}^{0}(\xi, \eta) \quad$ if $\quad \xi \eta>0$

(ii) $\lim _{\lambda \rightarrow \infty} \lambda q_{t}^{(\lambda)}(\xi, \eta)=\rho_{t}(\xi+|\eta|) \quad$ if $\eta<0<\xi$,

as follows. If $\xi \eta>0$, then as $\lambda \rightarrow \infty, E_{\xi}\left[e^{-\lambda \ell(t)} ; W_{t} \in d \eta, \sigma_{\{0\}}^{W}<t\right] / d \eta \rightarrow 0$, so that

$$
q_{t}^{(\lambda)}(\xi, \eta)=E_{\xi}\left[e^{-\lambda \ell(t)} ; W_{t} \in d \eta, \sigma_{\{0\}}^{W}>t\right] / d \eta+o(1)=\mathfrak{g}_{t}^{0}(\xi, \eta)\{1+o(1)\},
$$


showing (i). If $\eta<0<\xi$, (15.2) together with $\int_{0}^{t} \rho_{s}(\xi) \rho_{t-s}(|\eta|+u) d s=\rho_{t}(\xi+|\eta|+u)$ leads to

$$
\begin{aligned}
q_{t}^{(\lambda)}(\xi, \eta) & =\int_{0}^{t} \rho_{s}(\xi) E_{0}\left[e^{-\lambda \ell(t-s)} ; W_{t-s} \in d \eta\right] d s / d \eta \\
& =\int_{0}^{\infty} \rho_{t}(\xi+|\eta|+u) e^{-\lambda u} d u
\end{aligned}
$$

hence (ii) follows.

Now for $\eta, \zeta<0<\xi$ and $0<s<t$,

$$
\begin{aligned}
\lambda E_{\xi}\left[e^{-\lambda \ell(t)} ; W_{s} \in d \eta, W_{t} \in d \zeta\right] & =\lambda E_{\xi}\left[e^{-\lambda \ell(s)} \mathbf{1}_{d \eta}\left(W_{s}\right) E_{W_{s}}\left[e^{-\lambda \ell(t-s)} ; W_{t-s} \in d \zeta\right]\right] \\
& \sim \rho_{s}(\xi+|\eta|) \mathfrak{g}_{t-s}^{0}(\eta, \zeta) d \eta d \zeta
\end{aligned}
$$

disposing of the case $0=m<m^{\prime}=1$ (by taking $\xi=b, \zeta=-c$, and on using this result for $-c=\zeta, \eta<0<\xi$ and $0<s<t<T$,

$$
\begin{aligned}
& \lambda E_{b}\left[e^{-\lambda \ell(T)} ; W_{s} \in d \xi, W_{t} \in d \eta, W_{T} \in d \zeta\right] / d \xi d \eta d \zeta \\
& =\lambda E_{b}\left[e^{-\lambda \ell(s)} \mathbf{1}_{d \xi}\left(W_{s}\right) E_{W_{s}}\left[e^{-\lambda \ell(T-t)} ; W_{t-s} \in d \eta, W_{T-t} \in d \zeta\right]\right] / d \xi d \eta d \zeta \\
& \longrightarrow \mathfrak{g}_{s}^{0}(b, \xi) \rho_{t-s}(\xi+|\eta|) \mathfrak{g}_{T-t}^{0}(\eta,-c)
\end{aligned}
$$

$(\lambda \rightarrow \infty)$, showing the limit in (5.1) to agree with the corresponding density in (4.2) if $m=m^{\prime}=1$. The case $m^{\prime}<m=1$ is dealt with in a similar way and the general case by the double induction on $\left(m, m^{\prime}\right)$.

(B) Here is given a proof of (2.14). The proof is the same as for the Brownian case but we need to take care of asymmetry of the processes $Y^{\xi}$. Let $\xi>0$. Let $\sigma_{0}^{\xi, Y}$ be the first hitting time of zero for $Y^{\xi}$. Noting that the event $\sigma_{0}^{\xi, Y}>s$ entails $Y_{s}^{\xi}>0$ since $Y^{\xi}$ makes no negative jumps and $\mathfrak{p}_{s}^{\{0\}}(\xi, \eta)=\mathfrak{p}_{s}^{\{0\}}(-\eta,-\xi)$, we see

$$
\begin{aligned}
P\left[\sigma_{0}^{\xi, Y}>s+t\right] & =\int_{0}^{\infty} P\left[\sigma_{0}^{\xi, Y}>s, Y_{s}^{\xi} \in d \eta\right] P\left[\sigma_{0}^{\eta, Y}>t\right] \\
& =\int_{0}^{\infty} \mathfrak{p}_{s}^{\{0\}}(\xi, \eta) P\left[\sigma_{0}^{\eta, Y}>t\right] d \eta \\
& =\int_{t}^{\infty} d u \int_{0}^{\infty} \rho_{u}(\eta) \mathfrak{p}_{s}^{\{0\}}(-\eta,-\xi) d \eta,
\end{aligned}
$$

and differentiation yields $\rho_{s+t}(-\xi)=\int_{0}^{-\infty} \rho_{t}(\eta) \mathfrak{p}_{s}^{\{0\}}(\eta,-\xi) d \eta$ that is the same as (2.14).

\section{References}

[1] B. Belkin, An invariance principle for conditioned recurrent random walk attracted to a stable law, Zeit. Wharsch. Verw. Gebiete 21 (1972), 45-64.

[2] J. Bertoin, Lévy Processes, Cambridge Univ. Press, Cambridge (1996).

[3] J. Bertoin and R. A. Doney, On conditioning a random walk to stay positive, Ann. Probab, 22 (1994), 2152-2167.

[4] Q. Berger, Notes on random walks in the Cauchy domain of attraction. Probab. Theor. Rel. Field (2018). https://doi.org/10.1007/s00440-018-0887-0 
[5] P. Billingsley, Convergence of probability measures, John Wiley \& Sons, Ltd., New York 1968.

[6] F. Caravenna and L. Chaumont, An invariance principle for random walk bridges conditioned to stay positive, Electron. J. Probab. 18 (2013), no.60, 1-32.ISSN: 1883-6489 DOI: 18.1214/EJP.v18-2362

[7] R.A. Doney, Conditional limit theorems for asymptotically stable random walks, Z. Wahrsch. verw. Gebiete, 70 (1985), 351-360,

[8] R.A. Doney, Local behaviour of first passage probabilities, Probab. Theor. Rel. Fields, 152, (2012), 559-588.

[9] K. Itô and H.P. McKean, Jr, Diffusion processes and their sample paths. Springer, Berlin 1965.

[10] W. D. Kaigh, An invariance principle for random walk conditioned by a late return to zero. Ann Probab. 4 (1976), 115-121.

[11] D. Revuz and M. Yor, Continuous martingales and Brownian motion, 3ed ed., Springer, Berlin 1999.

[12] F. Spitzer, Principles of Random Walk, 2nd ed., Springer, Berlin 1976.

[13] K. Uchiyama, One dimensional lattice random walks with absorption at a point/on a half line, J. Math. Soc. Japan 63, (2011), 675-713.

[14] K. Uchiyama, One dimensional random walks killed on a finite set, Stoch. Proc. Appl. 127 (2017) 2864-2899.

[15] K. Uchiyama, Asymptotically stable random walks of index $1<\alpha<$ 2 killed on a finite set, Stochastic processes and their Applications (2019), https://doi.org/10.1016/j.spa.2019.02.006

[16] K. Uchiyama, Estimates of Potential functions of random walks on $\mathbb{Z}$ with zero mean and infinite variance and their applications. (preprint: available at: http://arxiv.org/abs/1802.09832.)

[17] K. Uchiyama, The Potential function and ladder variables of a recurrent random walk on $\mathbb{Z}$ with infinite variance. (preprint: available at: http://arxiv.org/abs/1805.03971.) 